\title{
4. Mauerwerk und Baumaterial
}

\subsection{Mauerproben und Erhaltungszustand}

Bereits Meyer-Plath/Schneider haben das Mauerwerk mehrerer Abschnitte stichwortartig charakterisiert und die Türme sowie die Kurtinen einzeln besprochen. ${ }^{655}$ Eine zusammenfassende Übersicht über den Mauercharakter einiger Abschnitte verdanken wir Foss. ${ }^{656}$ Metin und Zeynep Ahunbay veröffentlichten die Ergebnisse ihrer Untersuchung (Restaurierungen) an der Theodosianischen Landmauer zwischen Kurtine 1/2 und Turm 5. ${ }^{657}$ Eine umfassende und detaillierte Dokumentation, die das Mauerwerk der gesamten Anlage zwischen Marmarameer und Goldenem Horn erfaßt, wobei die Maße der Ziegel bzw. der Ziegelbänder, die zum Teil für eine relative Datierung hilfreich sein können, aufgenommen werden müßten, liegt noch nicht vor. Einerseits die immer wiederkehrenden Erdbeben, ${ }^{658}$ andererseits die an großen Partien der Mauer teilweise rasch durchgeführten Restaurierungen ${ }^{659}$ machen eine solche Dokumentation zu einem Desiderat, da sie für die topographischen Fragen die Grundlage bildet.

Im Rahmen meiner Arbeit wurde die gesamte Wehranlage vom Marmarameer bis zum Goldenen Horn - soweit möglich - mehrmals gründlich in Augenschein genommen. Es ist mir bewußt, daß eine unfassende Dokumentation nur mit Hilfe eines mit moderner Technik ausgerüsteten Teams geleistet werden kann. Daher erhebt der folgende Abschnitt auf keinen Fall den Anspruch, die bestehende Forschungslücke schließen zu wollen. Um möglichen Einwänden gegen die angewandte Untersuchungsmethode vorzubeugen, sollen hier einige Punkte ausdrücklich vermerkt werden: 1.) Es handelt sich bei dem untersuchten Objekt um eine mehrere Kilometer lange doppelte Mauer, d.h. jeweils mit einer Feld- und einer Stadtseite an der Haupt- und Vormauer. Besonders der Bereich innerhalb des Zwingers ist zum Teil schwer zugänglich oder gefährlich, weswegen während der Untersuchungen auf längere Aufenthalte in bestimmten Mauerabschnitten verzichtet werden mußte. Unvollständ-

655 Meyer-Plath/Schneider, Landmauer, passim.

656 Foss, Fortifications, 41-69; eine knappe Zusammenfassung auch bei Ward-Perkins, Notes, 66-67.

657 Ahunbay/Ahunbay, Land Walls, 227-239.

658 Zuletzt am 17. August 1999.

659 Für einen Überblick über die neueren Restaurierungsarbeiten und eine scharfe Kritik an einigen unkontrollierten Restaurierungsmaßnahmen siehe Ahunbay, Surlar, 74-80, bes. $79-80$. 
igkeiten sind daher unvermeidlich. ${ }^{660}$ 2.) Alle Maßaufnahmen erfolgten mit dem Zollstock, weshalb keine Genauigkeit auf den Millimeter erwartet werden darf. 3.) An den untersuchten Teilen wurden die Quader- und Ziegelmaße nur probeweise und zumeist in den unteren Bereichen der Mauerabschnitte aufgenommen, wobei sichtbare Unterschiede zu den oberen und innerhalb meiner Möglichkeiten erreichbaren Stellen notiert worden sind. 4.) Die Dimension der Mörtelschichten bzw. ihr Verhältnis zum umgebenden Mauerwerk können für eine relative Datierung hilfreich sein, weshalb ich bemüht war, auch die Mörtelhöhen aufzunehmen. Die Zusammensetzung der Mörtel bedarf jedoch chemischer Untersuchungen, um daraus Datierungskriterien gewinnen zu können. ${ }^{661}$

Da gegenwärtig mehrere Abschnitte der Mauer mit neuerem Material repariert werden und dabei an einigen Stellen ihre originale Substanz verlieren, soll die folgende Übersicht - soweit möglich - eine Vorstellung von den ursprünglichen Mauerwerten für künftige Arbeiten vermitteln. Die Besonderheiten des Mauerwerks werden im folgenden kurz beschrieben. Inschriften werden nur dann genannt, wenn darin ein Kaisername bzw. ein Datum erwähnt ist. $^{662}$

Verwendete Abkürzungen:

T: Turm

K: Kurtine

Die Buchstaben und Zahlen wie z. B. KO-1 beziehen sich auf die Katalognummern der dekorativen Spolien im 5. Abschnitt.

\subsubsection{Theodosianische Landmauer}

\subsubsection{Hauptmauer}

T. 1: Fünfseitig. Neuzeitlich restauriert (Abb. 112). In den unteren Partien größere Quader, sonst unterschiedliche Quaderlagen. Fünfschichtige Ziegelbänder. Ziegelbandhöhe $55 \mathrm{~cm}$, Ziegelbreite $36 \mathrm{~cm}$, Ziegelhöhe ca. $4,5 \mathrm{~cm}$, Mörtelhöhe $5,5 \mathrm{~cm}$. Reparaturinschrift: Konstantin und Basileios. ${ }^{663}$

660 Siehe dazu Dorso, Espace, 1-34: Der Autor versucht einen vollständigen Bericht über den Zustand der Landmauer zur geben, wobei er offenkundig etliche Mauerabschnitte gemieden haben muß.

661 Für eine chemische Untersuchung der Mörtel im Bereich von Turm 4 der Landmauer siehe Ersen/Acun/Gürdal/Güleç, Ancient Mortars, 165.

662 Für die Inschriften siehe im allgemeinen Meyer-Plath/Schneider, Landmauer, 123-144 (Schneider); MISN-Karte I; Mango, Inscriptions, 53. - Weitere Inschriftenfragmente wurden im Spolienkatalog (Abschnitt 5.10.) berücksichtigt.

663 Der hier erwähnte Basileios wird von Lietzmann, Landmauer 19, Nr. 1, und von MeyerPlath/Schneider, Landmauer, 123, Nr. 1, als Basileios II. angenommen (Schneider); vgl. auch Schneider, Yedikule, 199. - Foss, Inscriptions, 78-79, hält eine Identifizierung mit 
K. 1/2: Nebenöffnung. Neuzeitlich restauriert (Abb. 112). ${ }^{664}$ Sieben bis elf Quaderlagen, fünfschichtige Ziegelbänder. Ziegelbandhöhe $47 \mathrm{~cm}$.

T. 2: Achtseitig. Neuzeitlich restauriert. ${ }^{665}$ Fünfschichtige Ziegelbänder. Ziegelbandhöhe $47 \mathrm{~cm}$, Ziegelbreite $38 \mathrm{~cm}$, Ziegelhöhe $4 \mathrm{~cm}$, Mörtelhöhe 4,5 cm. - Im Turm (erstes Obergeschoß) mehrere Spolien K-1, K-2, K-14, TE-1, TE-2.

K. 2/3: Neuzeitlich restauriert. ${ }^{666}$ Wie T. 2.

T. 3: Rechteckig. Obere Hälfte und Südflanke abgestürzt. Neuzeitlich restauriert. ${ }^{667}$ Keine saubere Verbindung mit der Kurtine. Ziegelbandhöhe $48 \mathrm{~cm}$, Ziegelbreite $35 \mathrm{~cm}$, Ziegelhöhe 4 bis $5 \mathrm{~cm}$, Mörtelhöhe 4,5 bis $6,5 \mathrm{~cm}$. Mörtel mit Ziegelbrocken.

K. 3/4: Neuzeitlich restauriert. ${ }^{668}$ Fünfschichtige Ziegelbänder. Ziegelbandhöhe $57 \mathrm{~cm}$, Ziegelbreite 28 bis $36 \mathrm{~cm}$, Ziegelhöhe 4,5 bis $5 \mathrm{~cm}$, Mörtelhöhe 3,5 bis $7 \mathrm{~cm}$.

Basileios I. für wahrscheinlicher. Vgl. ders., Fortifications, 55. Maguire, Castle, 21-24, nimmt, von einem Gedicht ausgehend, an, daß Turm 1 im 10. Jahrhundert möglicherweise als Belvedere diente.

664 Zwischen Kurtine 1/2 und Turm 5 wurden in mehreren Kampagnen (1991 und 1993) unter Leitung von Metin und Zeynep Ahunbay von der İstanbul Teknik Universitesi Restaurierungen durchgeführt, wobei nicht nur die theodosianische Erstkonstruktion, sondern auch die späteren byzantinischen Reparaturen berücksichtigt worden sind, siehe Ahunbay/Ahunbay, Land Walls, 227-239, bes. 229-230: „Bricks measuring 37/38 by $37 / 38$ by $4,5-5$ centimeters had been used in the fifth century construction. However, within the wall core bricks 35/37 centimeter square were in abundance, they made for easier and quicker work. Smaller and/or thinner bricks were employed during Byzantine repairs and renovations. The original mortar was basically a mixture of lime, crushed brick and brick powder, the latter providing a hydraulic quality that simple lime mortar lacks. During the later Middle Ages, lime based mortars were used as well. For restoration the composition of mortar mixes was investigated and new mixes with matching colour and composition were developed by laboratory research." - Weitere Abschnitte der Mauern wurden hauptsächlich zwischen 1987 und 1994 von anderen Teams renoviert. Zur Zeit finden an verschiedenen Stellen wie Edirnekapı und Topkapı Restaurierungsarbeiten statt.

665 Ahunbay/Ahunbay, Land Walls, 230: „The fifth century fabric of the lower part of the western elevation was preserved, but the upper sections had been subject to alterations in the Middle Ages."

666 Ahunbay/Ahunbay, Land Walls, 232-233: „The slanting walls of the opening exhibit a different masonry technique from that used elsewhere. Bands of three brick courses alternate regularly with five courses of squared blocks, sheathing the exposed rubble core of the curtain wall on either side of the interruption. ... The regular alternation of brick and squared block courses suggest Palaiologen style."

667 Ahunbay/Ahunbay, 234: „Although the northern wall of the tower had large areas dating from the Middle Ages, the evidence indicated that the large cavity on this elevation was limited to a portion from the fifth century. During the repair, the original masonry techniques were used to rebuild the missing courses."

668 Ahunbay/Ahunbay, 234-235: „The upper levels of the wall had late Palaiologan repairs, which are distinguished by their roughly hewn stone blocks and insertion of some brick fragments into horizontal joints of the brick bands." 
T. 4: Achtseitig. Neuzeitlich restauriert. Sechs bis acht Quaderlagen und fünfschichtige Ziegelbänder. Ziegelbandhöhe $55 \mathrm{~cm}$, Ziegelbreite 34 bis $38 \mathrm{~cm}$, Ziegelhöhe 4,5 bis $5 \mathrm{~cm}$, Mörtelhöhe 6 bis $8 \mathrm{~cm}$, Stoßfuge $3 \mathrm{~cm}$. Reparaturinschrift: Romanos (Abb. 1). ${ }^{669}$

K. 4/5: Neuzeitlich restauriert. ${ }^{670}$ Fünfschichtige Ziegelbänder. Ziegelbandhöhe $47 \mathrm{~cm}$, Ziegelbreite $37 \mathrm{~cm}$, Ziegelhöhe 4,5 bis $6 \mathrm{~cm}$, Mörtelhöhe 4,5 bis $6 \mathrm{~cm}$.

T. 5: Rechteckig. Neuzeitlich restauriert. ${ }^{671}$ Südflanke abgestürzt (Erdbeben 1999). Ziegelbandhöhe: $50 \mathrm{~cm}$, Ziegelbreite 37 bis $38 \mathrm{~cm}$, Ziegelhöhe 4,5 bis $5,5 \mathrm{~cm}$, Mörtelhöhe 4,5 bis $6 \mathrm{~cm}$. - An der Südflanke I-6, ${ }^{672}$ an der Westfront PF-6.

K. 5/6: Fünfschichtige Ziegelbänder. Ziegelbandhöhe $49 \mathrm{~cm}$, Ziegelbreite 34 bis $37 \mathrm{~cm}$, Ziegelhöhe 4,5 bis $5 \mathrm{~cm}$, Mörtelhöhe 4,5 bis $5 \mathrm{~cm}$

T. 6: Achtseitig. In schlechtem Zustand. Fünfschichtige Ziegelbänder. Ziegelbandhöhe $48 \mathrm{~cm}$, Ziegelbreite $33 \mathrm{~cm}$, Ziegelhöhe 4,5 bis $5 \mathrm{~cm}$, Mörtelhöhe 4,5 bis $6,5 \mathrm{~cm}$.

K. 6/7: Fünfschichtige Ziegelbänder. Ziegelbandhöhe $44 \mathrm{~cm}$, Ziegelbreite $37 \mathrm{~cm}$, Ziegelhöhe $4 \mathrm{~cm}$, Mörtelhöhe 4 bis $5 \mathrm{~cm}$. Mörtel mit Ziegelbrocken.

Von hier bis Kurtine 8/9 nicht erhalten. ${ }^{673}$

K. 8/9: Fünfschichtige Ziegelbänder. Ziegelbandhöhe $45 \mathrm{~cm}$, Ziegelbreite 37 bis $38 \mathrm{~cm}$, Ziegelhöhe 4 bis $4,5 \mathrm{~cm}$, Mörtelhöhe 4 bis $4,5 \mathrm{~cm}$ (Abb. 126-128).

T. 9: Südpylon des Goldenen Tors. Mit Marmor verkleidet. Auf einigen Blöcken reliefierte Christogramme.

T. 10: Nordpylon des Goldenen Tors. Wie T. 9.

K. 10/11: Palaiologische und osmanische Reparaturen mit kleinen Quadern. An der Stadtseite untere Partien Quader mit Ziegeldurchschuß, obere Partien nur kleine Quader. Polychrome Nischenbögen (Abb. 110). Zum Teil Ziegelbänder. Ziegelbandhöhe $41 \mathrm{~cm}$, Ziegelbreite $37 \mathrm{~cm}$, Ziegelhöhe 4 bis $4,5 \mathrm{~cm}$, Mörtelhöhe ca. $4 \mathrm{~cm}$; zwischen den Ziegelbändern elf Quaderlagen, Höhe ca. 1,05 m, Quader länglich geschnitten.

669 Der hier erwähnte Kaiser Romanos wird im allgemeinen als Romanos III. Argyros (1028-1034) angenommen; siehe dazu Van Millingen, Walls, 102; Lietzmann, Landmauer, 10, Nr. 4; Meyer-Plath/Schneider, Landmauer, 124, Nr.4a, (Schneider); vgl. auch Schneider, Yedikule, 199. - Für eine Identifizierung mit Romanos II. (959-963) siehe Foss, Inscriptions, 80; ders., Fortifications, 56.

670 Ahunbay/Ahunbay, Land Walls, 237: „The fifth-century fabric could be followed up to the top of the wall. The eastern elevation, however, had been seriously damaged."

671 Ahunbay/Ahunbay, Land Walls, 238: „In the course of restoration, the vestibule was cleaned and the arch over the entrance repaired ... Another inscription block with letters AUTOKPATOP was unearthed during our work in 1992 in the peribolos between T. 4 and T. 5 (closer to the latter and now in the Archaeological Museums of Istanbul).“

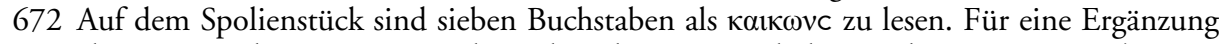
als Leon und Konstantin siehe Schneider/Meyer Plath, Landmauer, 71, 124, Nr.6 (Schneider). - Foss, Fortifications, 55, ergänzte zu Basileios und Konstantin.

$673 \mathrm{Zu}$ einer Inschrift am zerstörten Turm 7, die den Namen Leon und Konstantin erwähnt, siehe Meyer-Plath/Schneider, Landmauer, 124, Nr. 7 (Schneider); Schneider, Yedikule, 199. 
T. 11: Achtseitig. Osmanisch. Kleine bis mittelgroße regelmäßig geschnittene Quader (Abb. 3).

K. 11/12: Yedikulekapısı (Abb. 9). Südseite des Tors (Abb. 113-115) palaiologisch mit Quadern und Ziegeldurchschuß sowie osmanisch mit kleineren Quadern (siehe Abschnitt 2.2.2.1.). Nordseite zum Teil sechs Quaderlagen und fünfschichtige Ziegelbänder. Stadtseite oberhalb des Torbogens Quader mit breiten Lagefugen und Ziegeldurchschuß und P-8 (Abb. 10).

T. 12: Rechteckig. Neuzeitlich restauriert. Abgestürzt (Erdbeben 1999). Nur untere Teile erkennbar.

K. 12/13: Neuzeitlich restauriert.

T. 13: Achtseitig. Teilweise erhalten (Abb. 3). Abgestürzt (Erdbeben 1999). Neun Quaderlagen und fünfschichtige Ziegelbänder-

K. 13/14: Neuzeitlich restauriert. Quader mit engen Fugen und fünfschichtigen Ziegelbändern ( $41 \mathrm{~cm}$ hoch).

T. 14: Rechteckig. Westseite später (osmanisch) zurückgesetzt. An unteren Partien fünfschichtige Ziegelbänder zu erkennen. Ziegelbandhöhe $45 \mathrm{~cm}$, Ziegelbreite 38 bis $40 \mathrm{~cm}$, Ziegelhöhe 4 bis $4,5 \mathrm{~cm}$, Mörtelhöhe 4 bis $4,5 \mathrm{~cm}$.

K. 14/15: Wie Turm 14.

T. 15: Achtseitig. Im schlechten Zustand. Fünfschichtige Ziegelbänder. Ziegelbandhöhe $42 \mathrm{~cm}$, Ziegelbreite 29 bis $33 \mathrm{~cm}$, Ziegelhöhe 3 bis $3,5 \mathrm{~cm}$, Mörtelhöhe 4,5 bis $5 \mathrm{~cm}$.

K. 15/16: Neuzeitlich restauriert.

T. 16: Rechteckig. Obere Teile abgestürzt (Erdbeben 1999). Mehrere Quaderlagen und fünfschichtige Ziegelbänder. Ziegelbandhöhe $54 \mathrm{~cm}$, Ziegelbreite 36 bis $37 \mathrm{~cm}$, Ziegelhöhe 4,5 bis $5 \mathrm{~cm}$, Mörtelhöhe 4,5 bis $5 \mathrm{~cm}$. Kein sauberer Anschluß an die Kurtine. An der Südflanke Reparatur mit neunschichtigen Ziegelbändern.

K. 16/17: Schichtenmauerwerk. Neun bzw. elf Quaderlagen mit engen Fugen. Fünfschichtige Ziegelbänder.

T. 17: Polygonal. Südliche Hälfte abgestürzt. Neun bzw. elf Quaderlagen mit engen Fugen. Fünfschichtige Ziegelbänder. Ziegelbandhöhe 48 bis $52 \mathrm{~cm}$, Ziegelbreite 36 bis $37 \mathrm{~cm}$, Ziegelhöhe 4 bis $5 \mathrm{~cm}$, Mörtelhöhe 4 bis $5 \mathrm{~cm}$. Mörtel ist sehr rötlich (mit Ziegelbrocken) (Abb. 4).

K. 17/18: Neuzeitlich restauriert. Mauerwerk wie T. 17. Sauberer Anschluß an die flankierenden Türme.

T. 18: Rechteckig. Nördliche Flanke zur Hälfte abgestürzt (Abb. 50). Neun bzw. elf Quaderlagen. Fünfschichtige Ziegelbänder. Ziegelbandhöhe 49 bis $52 \mathrm{~cm}$, Ziegelbreite 35 bis $38 \mathrm{~cm}$, Ziegelhöhe 4 bis $5 \mathrm{~cm}$, Mörtelhöhe 5 bis $6 \mathrm{~cm}$ (aufgenommen am zweiten Ziegelband von unten). Besonders an den Verbindungsstellen mit der Kurtine ist theodosianisches Mauerwerk mit geringerer Ziegelbandbreite und engen Quaderfugen zu erkennen. Obere Teile der Westfassade repariert. Unregelmäßige Quader mit gele- 
gentlich verwendeten Ziegeln in den Stoß- und Lagefugen. Reparaturinschrift aus Ziegeln: Leon und Konstantin. ${ }^{67}$

K. 18/19: Teilweise erhalten. Untere Partien neuzeitlich intensiv restauriert. Sauberer Anschluß an die flankierenden Türme. ${ }^{675}$

T. 19: Rechteckig. Mittlerer vertikaler Teil der Westseite osmanisch repariert (Abb. 54). Sonst elf bzw. dreizehn Quaderlagen und fünfschichtige Ziegelbänder. Ziegelbandhöhe 48 bis $52 \mathrm{~cm}$, Ziegelbreite 30 bis $35 \mathrm{~cm}$, Ziegelhöhe 4,5 cm, Mörtelhöhe 5 bis $6 \mathrm{~cm}$. Fast gleiche Ziegelbandwerte wie T. 18. Reparaturinschrift aus Ziegeln: Leon und Konstantin. ${ }^{676}$ - An der Nordflanke in zugemauerter Pforte V-1.

K. 19/20: Teilweise erhalten. Untere Hälfte neuzeitlich restauriert. Sauberer Anschluß an die flankierenden Türme.

T. 20: Rechteckig. Acht bzw. elf Quaderlagen. Fünfschichtige Ziegelbänder. Ziegelbandhöhe $56 \mathrm{~cm}$, Ziegelbreite 35 bis $38 \mathrm{~cm}$, Ziegelhöhe 4,5 cm, Mörtelhöhe 5 bis $8 \mathrm{~cm}$. In unteren Partien geringe Stoßfugen zwischen den Quadern (Abb. 65).

K. 20/21: Untere Partien neuzeitlich restauriert. Sauberer Anschluß an die flankierenden Türme.

T. 21: Rechteckig. Fünfschichtige Ziegelbänder zu erkennen. In den unteren Partien große Marmorblöcke.

K. 21/22: Neuzeitlich restauriert.

T. 22: Südturm von Belgratkapı. Rechteckig. Neuzeitlich restauriert (Abb. 11, 68). ${ }^{677}$

T. 23: Nordturm von Belgratkapı. Rechteckig. Neuzeitlich restauriert (Abb. 11, 65, 68).

K. 23/24: Neuzeitlich restauriert.

T. 24: Rechteckig. Neuzeitlich intensiv restauriert (Abb. 65). Fünf Lagen flacher Blöcke. Fünfschichtige Ziegelbänder. Oberhalb der zweiten Steinlage ein Gesimsband. Darauf Schichtenmauerwerk mit unregelmäßigen und kleineren Quadern und breiteren Stoßund Lagefugen. Ziegelbandhöhe $45 \mathrm{~cm}$, Ziegelbreite $37 \mathrm{~cm}$, Ziegelhöhe $5 \mathrm{~cm}$, Mörtelhöhe $5 \mathrm{~cm}$. Paspatis zufolge Reparaturinschrift des Kaisers Konstantin. ${ }^{678}-$ PF-7.

K. 24/25: Neuzeitlich restauriert.

T. 25: Rechteckig. Teilweise neuzeitlich restauriert (Abb. 52, 65). Vier bis fünf Quaderlagen. Fünfschichtige Ziegelbänder. Untere Partien: Ziegelbandhöhe $48 \mathrm{~cm}$, Ziegelbreite $38 \mathrm{~cm}$, Ziegelhöhe $4 \mathrm{~cm}$, Mörtelhöhe 4 bis $4,5 \mathrm{~cm}$, Stoßfuge $3 \mathrm{~cm}$. Obere

674 Van Millingen, Walls, 98; Lietzmann, Landmauer, 21, Nr. 13; Meyer-Plath/Schneider, Landmauer, 127, Nr. 12 (Schneider).

$675 \mathrm{Zu}$ einer hier einst vorhandenen Inschrift von Leon und Konstantin siehe Schneider, Yedikule, 199.

676 Meyer-Plath/Schneider, Landmauer, 127, Nr. 13 (Schneider).

677 Zum alten Zustand der gesamten Toranlage vgl. Anm. 289.

678 Paspatis, Meletai, 57, las den Namen als Konstantin Palaiologos, äußerte aber Zweifel, ob er den Namen richtig entziffert hatte. Für eine kurze Diskussion und Lesung als Konstantin Porphyrogennetos siehe Schneider (Meyer-Plath/Schneider, Landmauer, 127, Nr. 15); vgl. auch Foss, Inscriptions, 85; ders., Fortifications, 56; 
Partien: Ziegelbandhöhe $47 \mathrm{~cm}$, Ziegelbreite $35 \mathrm{~cm}$, Ziegelhöhe $4 \mathrm{~cm}$, Mörtelhöhe $4,5 \mathrm{~cm}$. Reparaturinschrift aus Ziegeln: Leon und Konstantin (nicht erhalten). ${ }^{679}$

K. 25/26: Neuzeitlich restauriert.

T. 26: Rechteckig. An der Südflanke Ziegelbänder zu erkennen (Abb. 52, 65). An der Nordecke der Westfront unregelmäßige Quader mit sporadischem Ziegeldurchschuß. $\mathrm{KO}-5$.

K. 26/27: Nördliche Hälfte neuzeitlich restauriert. An der südlichen Hälfte fünfschichtige Ziegelbänder zu erkennen.

T. 27: Rechteckig. Neuzeitlich restauriert. Ursprünglich Schichtenmauerwerk mit breiten Fugen. An Nordfront enge Stoß- und Lagefugen bei den Quadern.

K. 27/28: Neuzeitlich restauriert. An Nordfront altes Mauerwerk mit breiten Fugen.

T. 28: Rechteckig. Teilweise osmanische, teilweise neuzeitliche Reparaturen zu erkennen. In schlechtem Zustand. Fünf Quaderlagen, fünfschichtige Ziegelbänder. Sehr breite Stoß- und Lagefugen. - K-6, K-7.

K. 28/29: Neuzeitlich restauriert.

T. 29: Rechteckig. Sehr beschädigt (Erdbeben 1999). An Nordflanke Schichtenmauerwerk zu erkennen. Ziegelbandhöhe $51 \mathrm{~cm}$, Ziegelbreite 31 bzw. $35 \mathrm{~cm}$, Ziegelhöhe 3 bzw. $4 \mathrm{~cm}$, Mörtelhöhe 6 bis $8 \mathrm{~cm}$, Stoßfugen 3 bzw. $4 \mathrm{~cm}$. An der Südflanke Cloissonnémauerwerk. Westseite mit unregelmäßigen Quadern.

K. 29/30: Neuzeitlich restauriert.

T. 30: Vierseitig. Mittlerer vertikaler Teil der Westseite neuzeitlich restauriert (Abb. 118). Fünf Quaderlagen und fünfschichtige Ziegelbänder fragmentarisch zu erkennen, sonst mit unregelmäßigen Quadern. Obere Partien mit unregelmäßigen Quadern. An Nordflanke unregelmäßige Quader mit gelegentlichem Ziegeldurchschuß.

K. 30/31: Nebenöffnung. Neuzeitlich restauriert (Abb. 118).

T. 31: Vierseitig. Sehr beschädigt (Erdbeben 1999). An der Südflanke fünfschichtige Ziegelbänder zu erkennen. In unteren Partien regelmäßige Quader und fünfschichtige Ziegelbänder. Ab dem zweiten Ziegelband eine Reihe Cloissonnémauerwerk. Obere Partien mit unregelmäßigen Quadern.

K. 31/32: Sieben bis zehn Quaderlagen. Fünfschichtige Ziegelbänder. Südliche Partie mit unregelmäßigen Quadern und gelegentlichem Ziegeldurchschuß.

T. 32: Rechteckig. Nordflanke beschädigt (Erdbeben 1999). Sechs Quaderlagen. Quader wiederverwendet. Fünf- bzw. sechsschichtige Ziegelbänder. Ziegelbandhöhe $61 \mathrm{~cm}$, Ziegelbreite $30 \mathrm{~cm}$, Ziegelhöhe 4 bis $4 \mathrm{~cm}$, Mörtelhöhe $6 \mathrm{~cm}$, Stoßfuge bis zu $5 \mathrm{~cm}$.

K. 32/33: Neuzeitlich restauriert.

T. 33: Rechteckig. In den unteren Partien sauber verlegte Quader mit engen Fugen und fünfschichtige Ziegelbänder. Obere Partien mit unregelmäßigen Quadern und sporadischem Ziegeldurchschuß.

679 Zur Inschrift siehe Lietzmann, Landmauer, 21, Nr. 14; Meyer-Plath/Schneider, Landmauer, 127, Nr. 16 (Schneider). 
K. 33/34: Neuzeitlich restauriert.

T. 34: Rechteckig. Mit unregelmäßigen Quadern restauriert. An Westfront fünfschichtige Ziegelbänder und sauber verlegte Quader mit engen Stoß- und Lagefuge. Reparaturinschrift Leon und Konstantin. ${ }^{60}$

K. 34/35: Neuzeitlich restauriert. Fünfschichtige Ziegelbänder fragmentarisch zu erkennen.

T. 35: Südturm von Silivrikapı. Sechsseitig. Neuzeitlich restauriert (Abb. 71) ${ }^{681}$ In den unteren Partien Quadern mit Ziegellagen. Oben unregelmäßige Quader mit Ziegeldurchschuß in den Lagefugen. An der Stadtseite Reparaturinschrift mit Jahresangabe 1438 (Abb. 72). ${ }^{682}$

T. 36: Nordturm von Silivrikapı. Sechseitig. Neuzeitlich restauriert (Abb. 12). In den unteren Partien fünfschichtige Ziegelbänder. Ziegelbandhöhe $52 \mathrm{~cm}$, Ziegelbreite $37 \mathrm{~cm}$, Ziegelhöhe 4,5 bis $5,5 \mathrm{~cm}$, Mörtelhöhe 4,5 bis $5 \mathrm{~cm}$, Stoßfuge $2 \mathrm{~cm}$. Inschriftband mit den Namen Basileios und Konstantin. ${ }^{63}$

K. 36/37: Neuzeitlich restauriert.

T. 37: Rechteckige Basis, polygonaler Schaft (Abb. 12, 73, 74). Basis mit zwölf Quaderlagen und fünfschichtigen Ziegelbändern. Schaft acht bis zwölf Quaderlagen und fünfschichtige Ziegelbänder. Inschriftband: Leon und Konstantin. ${ }^{684}$

K. 37/38: Untere Partien neuzeitlich restauriert. Obere Teile beschädigt.

T. 38: Vierseitig. In sehr schlechtem Zustand. Zieglbänder sind zu erkennen.

K. 38/39: In sehr schlechtem Zustand.

T. 39: Südturm des sog. 3. Nebentors. Rechteckig. Elf Quaderlagen und fünfschichtige Ziegelbänder. Ziegelbandhöhe 47 bis $50 \mathrm{~cm}$, Ziegelbreite 30 bis $37 \mathrm{~cm}$, Ziegelhöhe ca. $4 \mathrm{~cm}$, Mörtelhöhe 4,5 bis $6 \mathrm{~cm}$ (Abb. 76).

T. 40: Nordturm des sog. 3. Nebentors. Rechteckig (Abb. 77). Acht bzw. mehrere Quaderlagen mit fünfschichtigen Ziegelbändern. Ziegelbandhöhe $49 \mathrm{~cm}$, Ziegelbreite $38 \mathrm{~cm}$, Ziegelhöhe 3 bis $4 \mathrm{~cm}$, Mörtelhöhe 4,5 bis $7 \mathrm{~cm}$. Marmorblock mit Inschrift: Justinian II. (Abb. 199). ${ }^{685}$

K. 40/41: An der Stadtseite Quader mit Ziegeldurchschuß.

T. 41: Nach Meyer-Plath/Schneider befand sich kein Turm 41 in der Mitte des sog. Sigma. Da aber ein Turm in ihrer Numerierung berücksichtigt wurde, um eine spätere Korrektur auszuschließen, behalte ich T. 41 bei.

680 Meyer-Plath/Schneider, Landmauer, 128, Nr. 18 (Schneider), nicht erhalten.

681 Zum alten Zustand der gesamten Toranlage vgl. Anm. 291.

682 Lietzmann, Landmauer, 22, Nr. 17; Diskussion bei Meyer-Plath/Schneider, 128, Nr. 20 (Schneider). - Foss, Fortifications, 252, Abb. 17, lokalisiert die Inschrift an der Nordseite; vgl. Abb. 71.

683 Für unterschiedliche Lesungen siehe Van Millingen, Walls, 101; Lietzmann, Landmauer 23, Nr. 20; Meyer-Plath/Schneider, Landmauer, 129, Nr. 23 (Schneider).

684 Lietzmann, 23, Nr. 21; Meyer-Plath/Schneider, Landmauer, 130, Nr. 24 (Schneider).

685 Vgl. Anm. 118. 
K. 41/42: In sehr schlechtem Zustand, Schale größtenteils verloren (Abb. 53). Südliche Partie: Quader mit Ziegeldurchschuß und Ziegeln in den Stoßfugen. Nördliche Partie: Fünfschichtige Ziegelbänder zu erkennen. Ziegelbandhöhe $49 \mathrm{~cm}$, Ziegelbreite 35 bis $37 \mathrm{~cm}$, Ziegelhöhe 4,5 cm, Mörtelhöhe 4,5 bis $7 \mathrm{~cm}$.

T. 42: Rechteckig. In sehr schlechtem Zustand. Seitliche Flanken abgestürzt. Fünfschichtige Ziegelbänder zu erkennen. Ziegelbandhöhe $58 \mathrm{~cm}$, Ziegelbreite $35 \mathrm{~cm}$, Ziegelhöhe 4,5 cm, Mörtelhöhe $6 \mathrm{~cm}$, Stoßfuge $4 \mathrm{~cm}$.

K. 42/43. Nebenöffnung. ${ }^{686}$ Teilweise neuzeitlich restauriert (Abb. 53, 119). Untere Partien: Quaderlagen und fünfschichtige Ziegelbänder. Ziegelbandhöhe $58 \mathrm{~cm}$, Ziegelbreite $35 \mathrm{~cm}$, Ziegelhöhe 4 bis $4,5 \mathrm{~cm}$, Mörtelhöhe $6 \mathrm{~cm}$, Stoßfuge $3 \mathrm{~cm}$. Obere Partien: Quader mit ein- bzw. zweischichtigem Ziegeldurchschuß in den Lagefugen. Sauberer Anschluß an T. 42.

T. 43: Rechteckig. Westseite mit Quadern repariert, sonst fünfschichtige Ziegelbänder nur fragmentarisch zu erkennen (Abb. 53). Ziegelbandhöhe $47 \mathrm{~cm}$, Ziegelbreite $35 \mathrm{~cm}$, Ziegelhöhe $5 \mathrm{~cm}$, Mörtelhöhe 5 bis $6 \mathrm{~cm}$.

K. 43/44: Untere Partien neuzeitlich restauriert. Obere Teile im schlechten Zustand.

T. 44: Rechteckig. Quader und fünfschichtige Ziegelbänder. Ziegelbandhöhe $45 \mathrm{~cm}$, Ziegelbreite $36 \mathrm{~cm}$, Ziegelhöhe 4 bis $4,5 \mathrm{~cm}$, Mörtelhöhe 4 bis $4,5 \mathrm{~cm}$. Obere Partien mit unregelmäßigen Quadern repariert. An der Südflanke Holzanker.

K. 44/45: In schlechtem Zustand. Südflanke fragmentarisch erhalten. Fünfschichtige Ziegelbänder zu erkennen.

T. 45: Rechteckig. Südflanke abgestürzt. Vier bis neun Quaderlagen und fünfschichtige Ziegelbänder (Ziegelbreite $35 \mathrm{~cm}$ ). Fragmentarisch erhaltene Ziegelinschrift: Leon und Konstantin (Abb. 51), ${ }^{67}$ in unteren Bereichen ein weiterer Marmorblock (heute verschwunden): Leon, Konstantin und Eirene. ${ }^{68}$

K. 45/46: In sehr schlechtem Zustand.

T. 46: Fünfseitig. An unteren Partien große und saubere Blöcke. Sieben bis neun Quaderlagen. Fünfschichtiges Ziegelband. Mauerwerk wie T. $40{ }^{689}$ Oben spätere Reparaturen mit Quadern und zweilagigen Ziegeln.

K. 46/47: Nur untere Teile erhalten.

686 Vgl. Anm. 285.

687 Van Millingen, Walls, 99; Lietzmann, Landmauer, 23, Nr. 24; Meyer-Plath/Schneider, Landmauer, 131, Nr. 29a (Schneider).

688 Van Millingen, Walls, 99, zufolge sind hier Leon IV. und Konstantin V. gemeint (Zeitraum zwischen 776 und 780). Lietzmann, Landmauer, 23, Nr 25, und MeyerPlath/Schneider, Landmauer, 131, Nr.29b (Schneider) nahmen an, daß Leon III. und Konstantin IV. erwähnt seien und setzten die Inschrift in das Jahr 740/41; Foss, Fortifications, 54 datiert den Turm 775/780 (Leon IV. und Konstantin VI.).

$689 \mathrm{Zu}$ dem hier befindlichen Monogrammblock siehe Van Millingen, Walls, 100; Lietzmann, Landmauer, 23, Nr. 26; für die Zuschreibung an Kaiser Justinian II. siehe MeyerPlath/Schneider, Landmauer, 131, Nr. 30 (Schneider); vgl. auch Foss, Fortifications, 53 (Zuschreibung an Kaiser Artemius); für eine Nahaufnahme der Blöcke siehe Tunay, Findings, 221, Abb. 5. 
T. 47: Rechteckig. Westseite fragmentarisch erhalten. Mauerwerk wie T. 45. Rest einer Ziegelinschrift. ${ }^{690}$

K. 47/48: Untere Teile fragmentarisch erhalten.

T. 48. Rechteckig. Neun bis elf Quaderlagen. Fünf- bzw. sechsschichtige Ziegelbänder. Ziegelinschrift: Leon und Konstantin. ${ }^{691}$

K. 48/49: Neuzeitlich restauriert.

T. 49: Fünfseitig. Neuzeitlich restauriert.

K. 49/50: Neuzeitlich restauriert.

T. 50: Südturm von Mevlevihanekapı. Rechteckig. Neuzeitlich restauriert. ${ }^{692}$ Längliche Blöcke.

T. 51: Nordturm von Mevlevihanekapı. Rechteckig. Neuzeitlich restauriert. Längliche Blöcke (Abb. 14).

Spätere Verkleinerungsteile des Tors: Ziegelbreite 39 bis $40 \mathrm{~cm}$, Ziegelhöhe $4 \mathrm{~cm}$, Mörtelhöhe $7 \mathrm{~cm}$.

K. 51/52: Quader mit Ziegeldurchschuß in den Lagefugen. Polychrome Bögen (Abb. 190). - P-5.

T. 52: Rechteckig. Nur Westseite fragmentarisch erhalten. Mittelgroße Quader.

K. 52/53: Neuzeitlich restauriert.

T. 53: Achtseitig. Neuzeitlich restauriert.

K. 53/54: Neuzeitlich restauriert.

T. 54: Rechteckig. Neuzeitlich restauriert. Sechs Quaderlagen. Fünfschichtige Ziegelbänder. Ziegelinschrift ohne Namen (Abb. 5). ${ }^{63}$

K. 54/55: Neuzeitlich restauriert.

T. 55: Rechteckig. Neuzeitlich restauriert. Wiederverwendete Quader und fünfschichtige Ziegelbänder. Ziegelinschrift: Leon und Konstantin. ${ }^{694}$

K. 55/56: Neuzeitlich restauriert.

690 Meyer-Plath/Schneider, Landmauer, 131, Nr.31 (Schneider); ebd., 77, wird der Turm von Meyer-Plath 740/41 datiert; vgl. Foss, Inscriptions, 81; für eine Datierung in die Zeit zwischen 775/780 siehe auch ders., Fortifications, 54.

691 Meyer-Plath/Schneider, Landmauer, 132, Nr. 32 (Schneider); ebd., 77 wird der Turm 740/41 datiert (Meyer-Plath). Foss, Fortifications, 54, zufolge nennt die Inschrift möglicherweise die Kaiser Leon IV. und Konstantin VI., weshalb er die Inschrift im Zeitraum 775/780 ansetzt; vgl. ders., Inscriptions, 83.

692 Für die Inschriften siehe Lietzmann, 24, Nr.27-31; Meyer-Plath/Schneider, Landmauer, 132, Nr.33a/b; $\$ 4$, Nr.34-36. - Bei Nr.36 handelt es sich um Inschriften, die auf drei Blöcken angebracht sind, vorauf der Name Konstantin vorkommt (Schneider); vgl. Mango, Inscriptions, 54, Nr. 11; siehe auch Ševčenko, Inscription, 1-8.

693 Zur Diskussion siehe Meyer-Plath/Schneider, Landmauer, 134, Nr. 37 (Schneider); ebd., 77 von Meyer-Plath 740/41 datiert.

694 Meyer-Plath/Schneider, Landmauer, 134, Nr. 38 (Schneider). 
T. 56: Achtseitig. Neun bis elf Quaderlagen. Fünfschichtige Ziegelbänder. Ziegelbandhöhe $49 \mathrm{~cm}$, Ziegelbreite $35 \mathrm{~cm}$, Ziegelhöhe $4,5 \mathrm{~cm}$, Mörtelhöhe 4,5 bis $5,5 \mathrm{~cm}$. Ziegelinschrift: Leon und Konstantin. ${ }^{695}$

K. 56/57: In sehr schlechtem Zustand.

T. 57: Rechteckig. Fünf bis zwölf Quaderlagen (kleinere Quader). Fünfschichtige Ziegelbänder. Ziegelbandhöhe $47 \mathrm{~cm}$, Ziegelbreite $34 \mathrm{~cm}$, Ziegelhöhe 4,5 cm, Mörtelhöhe 4,5 bis $7 \mathrm{~cm}$. Ziegelinschrift: Konstantinos Porphyrogennetos. ${ }^{696}$

K. 57/58: Neuzeitlich restauriert.

T. 58: Rechteckig. Nur beide Flanken fragmentarisch erhalten.

K. 58/59: In sehr schlechtem Zustand.

T. 59: Südturm vom Romanos-Tor. Rechteckig. Nur Nordflanke erhalten. Wiederverwendete Quader mit Ziegeldurchschuß (Abb. 83). ${ }^{697}$

T. 60: Nordturm vom Romanos-Tor. Rechteckig. Stadtseite neuzeitlich restauriert. Fünfschichtige Ziegelbänder zum Teil zu erkennen.

K. 60/61: Bei Anlage der Millet Caddesi abgebrochen.

T. 61: Rechteckig. Neuzeitlich restauriert. In unteren Partien große Quader mit engen Stoß- und Lagefugen. Fünfschichtige Ziegelbänder. Obere Teile mit unregelmäßigen Quadern repariert. An der Stadtseite fünfschichtige Ziegelbänder fragmentarisch zu erkennen, sonst Reparatur mit unregelmäßigen Quadern. An der Stadtseite osmanisches Spitzbogentor. - GFA-17 (Abb. 197).

K. 61/62: Neuzeitlich restauriert. Acht Quaderlagen. Fünf Ziegelschichten.

T. 62: Rechteckig. Unterschiedlich große Quaderlagen. Fünfschichtige Ziegelbänder. Ziegelbandhöhe $46 \mathrm{~cm}$, Ziegelbreite $37 \mathrm{~cm}$. Obere Teile mit unregelmäßigen Quadern repariert.

K. 62/63: Neuzeitlich restauriert. Zur Zeit wieder unter Restaurierung. Ziegelbänder zu erkennen. In unteren Partien geringfügig Quader. In oberen Bereichen unregelmäßige Quader mit gelegentlichem Ziegeldurchschuß.

T. 63: Achtseitig. Stadtseitiger Eingang in sehr schlechtem Zustand. Schichtenmauerwerk. Elf Quaderlagen. Fünf- bzw. siebenschichtige Ziegelbänder mit breiteren Stoßund Lagefugen. Ziegelinschrift ohne Kaisernamen. ${ }^{698}$ Obere Teile mit Quadern repariert, darauf KO-3 und KO-4 (Abb. 49, 191).

695 Meyer-Plath/Schneider, Landmauer, 134, Nr.39 (Schneider).

696 Der hier erwähnte Kaiser wird im allgemeinen mit Konstantin VII. Porphyrogennetos in Verbindung gebracht, siehe Meyer-Plath/Schneider, Landmauer, 134, Nr. 40 (Schneider). Lietzmann, Landmauer, 25, Nr.33, nahm an, daß hier auch der Name Leon vorhanden war. Foss, Fortifications, 55, hielt eine Identifizierung mit Konstantin VI. ebenfalls für möglich; vgl. ders., Inscriptions, 69.

697 Für die Inschriften am Vortor siehe Meyer-Plath/Schneider, Landmauer, 135, Nr.41 (Schneider); am Haupttor Asutay, Romanos-Tor, 1-4, Taf. II, 2-3.

698 Schneider (Meyer-Plath/Schneider, Landmauer, 136, Nr. 42b) datierte den Turm 740/41 und plazierte die Inschrift an Turm 62; Meyer-Plath, ebd., 78, mit richtigen Angaben. 
K. 63/64: Neuzeitlich restauriert. Zur Zeit wieder unter Restaurierung. Acht bis zehn Quaderlagen. Fünfschichtige Ziegelbänder. An der Stadtseite obere Partien mit unregelmäßigen Quadern repariert.

T. 64: Rechteckig. Zur Zeit Wiederherstellung mit neuem Material.

K. 64/65: Neuzeitlich restauriert. Sechs bzw. sieben Quaderlagen. Fünfschichtige Ziegelbänder (Abb. 89). - TE-7.

T. 65: Südturm von Topkapı. Rechteckig. Neuzeitlich restauriert. Große längliche Blöcke (Abb. 90).

T. 66: Nordturm von Topkapı. Rechteckig. Neuzeitlich restauriert. Fünfschichtige Ziegelbänder (Abb. 16, 89). - Mehrere Spolien K-8, K- 17, K-18.

K. 66/67: Neuzeitlich restauriert. Stadtseite Quader mit ein- bzw. zweischichtigem Ziegeldurchschuß in den Lagefugen.

T. 67: Rechteckig. In sehr schlechtem Zustand. In den unteren Partien geringfügig Quader teilweise zu erkennen.

K. 67/68: Mehrere unterschiedliche Reparaturen. In unteren Bereichen (Stadtseite) Ziegelbandhöhe $51 \mathrm{~cm}$, Ziegelbreite $37 \mathrm{~cm}$, Ziegelhöhe 4 bis $5 \mathrm{~cm}$, Mörtelhöhe 4 bis $5 \mathrm{~cm}$.

T. 68: Rechteckig. Besonders Stadtseite in sehr schlechtem Zustand. Elf Quaderlagen. Fünfschichtige Ziegelbänder. Ziegelbandhöhe $41 \mathrm{~cm}$, Ziegelbreite $37 \mathrm{~cm}$.

K. 68/69: Nicht erhalten.

T. 69: Rechteckig. Mehrere Reparaturen teils mit Quadern, teils nur mit Ziegeln. Fünfschichtige Ziegelbänder zu erkennen.

K. 69/70: In sehr schlechtem Zustand. Ziegelbänder nur fragmentarisch zu erkennen.

T. 70: Rechteckig. Neuzeitlich renoviert. Vier bis zehn Quaderlagen und fünfschichtige Ziegelbänder.

K. 70/71: In den unteren Partien Quader mit engen Stoß- und Lagefugen. Obere Teile Quader mit Ziegeldurchschuß in den Stoß- und Lagefugen. An der Stadtseite große Pfeiler als Spolien.

T. 71: Rechteckig. In den unteren Partien Quader mit engen Stoß- und Lagefugen. Fünfschichtige Ziegelbänder. Ziegelbandhöhe $45 \mathrm{~cm}$, Ziegelbreite $37 \mathrm{~cm}$, Ziegelhöhe $4 \mathrm{~cm}$, Mörtelhöhe $4 \mathrm{~cm}$. Obere Partien mit unregelmäßigen Quadern.

K. 71/72: Nur teilweise erhalten. Quader mit engen Stoß- und Lagefugen und fünfschichtige Ziegelbänder. Das Mauerwerk wie T. 71. Obere Teile mit unregelmäßigen Quadern repariert.

T. 72: Bei Anlage der Vatan Caddesi abgerissen.

K. 72/73: Wie T. 72 .

T. 73: Rechteckig. In sehr schlechtem Zustand. Nur in unteren Partien fünfschichtige Ziegelbänder zu erkennen. Ziegelbandhöhe $51 \mathrm{~cm}$, Ziegelbreite $35 \mathrm{~cm}$, Ziegelhöhe 3 bis $3,5 \mathrm{~cm}$, Mörtelhöhe 3 bis $8 \mathrm{~cm}$.

K. 73/74: Nur in unteren Teilen fünfschichtige Ziegelbänder zu erkennen. Obere Teile unregelmäßige Quader mit ein bis zwei Ziegelreihen in den Lagefugen. 
T. 74: Rechteckig. In den unteren Partien Ziegelbänder. Ziegelbandhöhe $51 \mathrm{~cm}$, Ziegelbreite 35 bis $37 \mathrm{~cm}$, Ziegelhöhe $5 \mathrm{~cm}$, Mörtelhöhe 5 bis $7,5 \mathrm{~cm}$. Obere Teile Quader mit Ziegeldurchschuß.

K. 74/75: Neuzeitlich restauriert. In unteren Partien fünfschichtige Ziegelbänder fragmentarisch zu erkennen (Ziegelbandhöhe $40 \mathrm{~cm}$ ). Obere Partien Quader mit ein bis zwei Ziegelschichten in den Lagefugen.

T. 75 (Sulukule): Rechteckig. Fünf bis sechs Quaderlagen und fünfschichtige Ziegelbänder. Ziegelbandhöhe $56 \mathrm{~cm}$, Ziegelbreite $38 \mathrm{~cm}$, Ziegelhöhe 3,5 bis $4,5 \mathrm{~cm}$, Mörtelhöhe 5 bis $7 \mathrm{~cm}$ (Abb. 55).

K. 75/76: Nördliche Partie und gesamte Stadtseite neuzeitlich restauriert. Südpartie mit unregelmäßigen Quadern mit Ziegeldurchschuß in den Stoßfugen sowie gelegentlich in den Lagefugen.

T. 76: Nur Nordflanke erhalten. Fünfschichtige Ziegelbänder zu erkennen. Ziegelbandhöhe $49 \mathrm{~cm}$, Ziegelbreite 35 bis $37 \mathrm{~cm}$, Ziegelhöhe 3 bis 4,5 cm, Mörtelhöhe $6 \mathrm{~cm}$. Rötlicher Mörtel mit vielen Ziegelbrocken.

K. 76/77: Teilweise erhalten. Stadtseite neuzeitlich renoviert.

T. 77: Südturm von Sulukulekapı. Rechteckig. Nur Nordflanke erhalten. Fünfschichtige Ziegelbänder (Abb. 17). ${ }^{699}$

T. 78: Nordturm von Sulukulekapı. Rechteckig. Südflanke erhalten. Untere Partien große Blöcke. Obere Teile Quader mit Ziegeldurchschuß in den Lagefugen. Ein $50 \mathrm{~cm}$ hoher Sockel um die Türme und das Torhaus (Abb. 17).

K. 78/79: Zum Teil Schale verloren, sonst fünfschichtige Ziegelbänder.

T. 79: Rechteckig. Nur Westseite teilweise erhalten. In unteren Partien fünfschichtige Ziegelbänder zu erkennen. Ziegelbandhöhe $43 \mathrm{~cm}$, Ziegelbreite $34 \mathrm{~cm}$. Obere Teile mit unregelmäßigen Quadern mit Ziegeln in den Stoßfugen repariert (Abb. 95).

K. 79/80: Teilweise erhalten. Unregelmäßige Quader.

T. 80: Nicht erhalten.

K. 80/81: In unteren Partien fünfschichtige Ziegelbänder zu erkennen. Ab dem zweiten Band unregelmäßige Quader mit Ziegeldurchschuß in den Lagefugen. - GFA-4, GFA10, I-7 (Abb. 188). - Meyer-Plath/Schneider, Faltplan 3, setzten hier noch einen T. 80 an, der nicht erhalten ist. Ich behalte ihre Numerierung bei.

T. 81: Nicht erhalten.

K. 81/82: Neuzeitlich restauriert. In unteren Bereichen ein fünfschichtiges Ziegelband. Stadtseite: Ziegelbandhöhe 48 bzw. $54 \mathrm{~cm}$, Ziegelbreite 32 bis $35 \mathrm{~cm}$, Ziegelhöhe 3 bis $4 \mathrm{~cm}$, Mörtelhöhe 6,5 bis $9 \mathrm{~cm}$. Feldseite: Ziegelbandhöhe $55 \mathrm{~cm}$, Ziegelbreite 32 bis $35 \mathrm{~cm}$, Ziegelhöhe 3 bis $4 \mathrm{~cm}$, Mörtelhöhe 6,5 bis $8 \mathrm{~cm}$. Sonst teils mit unregelmäßigen Quadern (Südpartie), teils mit Quadern mit Ziegeldurchschuß in den Lagefugen repariert (Nordpartie, Abb. 104, 105). - Kleinere Spolien, darunter I-8.

699 Für die Inschrift am Torsturz siehe Lietzmann, Landmauer, 26, Nr.36; Meyer-Plath/ Schneider, Landmauer, 136, Nr. 44 (Schneider). 
T. 82: Nicht erhalten. Turmeingang dient heute als Durchgang in die dahinter liegenden Straße (Abb. 104, 105).

K. 82/83: Durch moderne Straße unterbrochen. Am Südteil unten fünfschichtige Ziegelbänder und oben Quader mit Ziegeldurchschuß. Am Nordteil unregelmäßige Quader und fünfschichtige Ziegelbänder. Stadtseite: Ziegelband 51 bzw. $62 \mathrm{~cm}$, Ziegelbreite $31-42 \mathrm{~cm}$, Ziegelhöhe 2,5 bis $6 \mathrm{~cm}$, Mörtelhöhe 6 bis $10 \mathrm{~cm}$ (Abb. 106). Feldseite: Ziegelbandhöhe 44 bzw. $55 \mathrm{~cm}$, Ziegelbreite 32 bis $35 \mathrm{~cm}$, Ziegelhöhe 3 bis $4 \mathrm{~cm}$, Mörtelhöhe 6 bis 7,5 cm. Sauberer Verband mit Turm 83 (Abb. 107, 108).

T. 83: Rechteckig. Kein Eingang in der Stadtseite. Acht bis zwölf Quaderlagen. Fünfschichtige Ziegelbänder. Ziegelbandhöhe $55 \mathrm{~cm}$, Ziegelbreite 32 bis $35 \mathrm{~cm}$, Ziegelhöhe 3,5 bis $5 \mathrm{~cm}$, Mörtelhöhe 6 bis $7,5 \mathrm{~cm}$. Obere Partien sind mit unregelmäßigen Quadern und gelegentlich verwendeten Ziegeln repariert (Abb. 109). - An der Westfront einige Pfeiler mit Soffittenmuster und GFA-5, GFA-6 (Abb. 195, 196).

K. 83/84: Mauerwerk wie K. 82/83.

T. 84: Rechteckig. In sehr schlechtem Zustand. Nordflanke beschädigt. An Westseite sieben bis zehn Quaderlagen (unregelmäßig) mit breiteren Stoß- und Lagefugen und fünf- bis sechsschichtige Ziegelbänder. Ziegelbandhöhe $55 \mathrm{~cm}$, Ziegelbreite $35 \mathrm{~cm}$, Ziegelhöhe 3,5 bis $4 \mathrm{~cm}$, Mörtelhöhe 5 bis $7 \mathrm{~cm}$, Stoßfugen 5 bis $7 \mathrm{~cm}$. Obere Partien mit unregelmäßigen Quadern repariert, gelegentlich Ziegel in den Stoß- und Lagefugen. Südflanke mit unregelmäßigen Quadern repariert. - An der Westfront KO-11, KO-12, KO-13, an der Südflanke GFA-12 (Abb. 110, 111, 198).

K. 84/85: Unterste Partie im Schutt. Sieben Quaderlagen (sauber und scharfkantig, 140 x $60 \mathrm{~cm}$ bis $70 \times 80 \mathrm{~cm}$ ). Siebenschichtiges Ziegelband. Drei Quaderlagen $(47 \times 50 \mathrm{~cm})$ und aufgehendes Ziegelmauerwerk.

T. 85: Nicht erhalten.

K. 85/86: Mauerwerk an der Feldseite wie K. 84/85. An der Stadtseite mehrere Reparaturen. Fünfschichtige Ziegelbänder (Abb. 98).

T. 86: Südturm von Edirnekapı. Sechsseitig. Neuzeitlich intensiv restauriert. Ziegelbandhöhe ca. $65 \mathrm{~cm}$, Ziegelbreite $35 \mathrm{~cm}$, Ziegelhöhe 4,5 bis $5 \mathrm{~cm}$, Mörtelhöhe 6 bis $8 \mathrm{~cm}$. Stadtseitige Kurtine: Ziegelbandhöhe $62 \mathrm{~cm}$, Ziegelbreite $35 \mathrm{~cm}$, Ziegelhöhe 4,5 bis $6 \mathrm{~cm}$, Mörtelhöhe 6 bis $8 \mathrm{~cm}$ (Abb. 97).

T. 87: Nordturm von Edirnekapı. Fünfseitig. Neuzeitlich intensiv restauriert. Stadtseitige Verstärkung: Vierlagige wiederverwendete unregelmäßige Quader, vier- bis fünfschichtige Ziegelbänder. Ziegelbandhöhe $40 \mathrm{bzw} .51 \mathrm{~cm}$, Ziegelbreite $37 \mathrm{~cm}$, Ziegelhöhe 3 bis $6 \mathrm{~cm}$, Mörtelhöhe 5 bis $7 \mathrm{~cm}$ (Abb. 96).

K. 87/88: An der Feldseite wie K. 84/85, unterste Partie noch zu erkennen. Zwei Quaderlagen, siebenschichtiges Ziegelband, sieben Quaderlagen, siebenschichtiges Ziegelband, zwei Quaderlagen, darauf Ziegelmauerwerk (Abb. 102). An der Stadtseite mehrere Reparaturen.

T. 88: Rechteckig. Obere Teile und Westseite beschädigt. Mauerwerk wie K. 84/85 (Abb. 56, 102).

K. 88/89: Neuzeitlich restauriert. Mauerwerk wie K. 84/85 (Abb. 56, 102). 
T. 89: Rechteckig. Neuzeitlich restauriert. ${ }^{700}$

K. 89/90: Bei Anlage der Fevzi Paşa Caddesi abgerissen. An der Kurtine befand sich einst eine halbrunde Bastion.

T. 90: Achtseitig. Quaderwerk. In den unteren Partien ein fünfschichtiges Ziegelband zu erkennen.

K. 90/91: Neuzeitlich renoviert. Fünfschichtige Ziegelbänder zu erkennen. Mittlere Partie mit unregelmäßigen Quadern mit Ziegeldurchschuß renoviert.

T. 91: Rechteckig. Neuzeitlich restauriert. In unteren Partien fünfschichtige Ziegelbänder zu erkennen. Obere Partien unregelmäßige Quader mit gelegentlichem Ziegeldurchschuß in den Lagefugen. An Nord- und Südseite befanden sich einst Inschriftreste als Spolien. ${ }^{701}$

K. 91/92: Neuzeitlich restauriert. - An der Stadtseite K-9, K-13.

T. 92: Rechteckig. Fünfschichtige Ziegelbänder zu erkennen. Obere Teile sind mit unregelmäßigen Quadern repariert.

K. 92/93: Neuzeitlich restauriert.

T. 93: Rechteckig. Drei bzw. fünf Quaderlagen. Fünfschichtige Ziegelbänder. Ziegelbandhöhe $47 \mathrm{~cm}$, Ziegelbreite 27 bis $35 \mathrm{~cm}$, Ziegelhöhe $4 \mathrm{~cm}$, Mörtelhöhe 4,5 cm. Zur Zeit unter Restaurierung (Abb. 57). ${ }^{702}$

K. 93/94: Neuzeitlich restauriert.

T. 94: Rechteckig. Südflanke abgestürzt. Ohne Schieß- und Lichtscharten. Westfront: In unteren Partien große Blöcke, acht bis zehn Quaderlagen (abwärts kleinere). Drei- bis fünfschichtige Ziegelbänder. Ziegelbandhöhe $44 \mathrm{~cm}$, Ziegelbreite $37 \mathrm{~cm}$, Ziegerhöhe $4 \mathrm{~cm}$, Mörtelhöhe 3 bis $7 \mathrm{~cm}$, Stoßfuge $2,5 \mathrm{~cm}$. Nordflanke: Ziegelbandhöhe $55 \mathrm{~cm}$, Ziegelbreite $32 \mathrm{~cm}$, Ziegelhöhe $5 \mathrm{~cm}$, Mörtelhöhe $7 \mathrm{~cm}$. Obere Partien mit Quadern und Ziegeldurchschuß in den Lagefugen renoviert.

K. 94/95: Nur untere Teile erhalten. Mauerwerk wie T. 94.

T. 95: Rechteckig. Westfront mit drei bis fünf Quaderlagen und fünfschichtigen Ziegelbänder. Breitere Fugen zwischen den Quadern. Südflanke mit Quadern und Ziegeldurchschuß in den Lagefugen renoviert. Teilweise Cloissonnémauerwerk zu erkennen. $^{703}$

K. 95/96: Nur untere Teile erhalten.

T. 96: Rechteckig. Drei bis vier Quaderlagen. Fünfschichtige Ziegelbänder zu erkennen. Überwiegend unregelmäßige Quader mit Ziegeldurchschuß in den Lagefugen.

700 Wegen der schmalen Quader datierte Meyer-Plath (Meyer-Plath/Schneider, Landmauer, 80) den Turm um 700.

701 Meyer-Plath/Schneider, Landmauer, 138, Nr. 51 und 52 (Schneider).

702 Für eine verschwundene Inschrifttafel siehe Meyer-Plath/Schneider, 138, Nr.53 (Schneider).

703 Die auf den Quadern eingeritzte Inschrift ist nicht zu sehen, siehe Meyer-Plath/ Schneider, Landmauer, 139, Nr. 54 (Schneider). 


\subsubsection{Vormauer}

Vortürme 1a bis 3a nicht erhalten. ${ }^{704}$

T. 4a: Vierseitig. Nordflanke Quader mit zwei Ziegeldurchschüssen in den Lagefugen, sonst unregelmäßige Quader. Die oberen Verbindungsecken mit den Kurtinen aus zwölf Ziegelschichten.

K. 4a/5a: An unteren Partien kleine Quader und Ziegelbänder. Mit unregelmäßigen Quadern repariert. ${ }^{705}$

T. 5a: Halbrund. Quaderwerk. Ein Gesimsband. An den Flanken Ziegelbänder zu erkennen. Die oberen Verbindungsecken mit den Kurtinen aus zwölf Ziegelschichten.

K. 5a/6a: Untere Teile regelmäßige Quader. Fünfschichtige Ziegelbänder. Ziegelbandhöhe $49 \mathrm{~cm}$, Ziegelbreite $34 \mathrm{~cm}$, Ziegelhöhe $4 \mathrm{~cm}$, Mörtelhöhe $4 \mathrm{~cm}$.

K. 9a/10a: Vorwerk des Goldenen Tors (siehe Abschnitt 2.1.5.)

T. 10a: Nicht erhalten.

K. 10a/11a: Nicht erhalten.

T. 11a: Nicht erhalten. ${ }^{706}$

K. $11 \mathrm{a} / 12 \mathrm{a}$ : Repariert.

T. 12a: Vierseitig. Unregelmäßige Quader und gelegentlicher Ziegeldurchschuß in den Stoß- und Lagefugen. Inschrifttafel: Ioannes Palaiologos und Datum 1443/44 (nicht erhalten) (Abb. 3).

K. 12a/13a: Südliche Partien Quader mit Ziegeldurchschuß und Ziegeln in den Lagefugen repariert.

T. 13a: Halbrund. Quader mit Ziegeldurchschuß bzw. Kalksteinband (Abb. 3, 62).

K. 13a/14a: Sechs Quaderlagen. Drei schmale Quaderreihen, die Ziegelband ersetzen. Dreischichtige Ziegelbänder (Ziegelbreite $35 \mathrm{~cm}$ ).

T. 14a: Vierseitig. In sehr schlechtem Zustand.

K. 14a/15a: In sehr schlechtem Zustand.

T. 15a: Nicht erhalten.

704 Bereits auf der 1939 von Pervititch angefertigten Karte sind die Türme 1a, 2a nicht mehr eingetragen. Dagegen stand der rechteckige Turm 3a noch aufrecht, siehe Sigorta Haritalar1, 200. Turm 2a enthielt einst eine Reparaturinschrift mit dem Kaisernamen Ioannes Palaiologos, siehe dazu Paspatis, Meletai, 59; Van Millingen, Walls, 104; Lietzmann, Landmauer, 19, Nr. 3. - Nach Meyer-Plath/Schneider, Landmauer, 124, Nr. 5, könnte diese Inschrift mit der von Wulff, Katalog, unter Nr. 184, angegebenen Inschrift identisch sein (Schneider); siehe auch Schneider, Yedikule, 199.

705 Für einen nicht mehr erhaltenen Inschriftrest siehe Meyer-Plath/Schneider, Landmauer, 124, Nr. 5 (Schneider); Schneider, Yedikule, 199.

706 Für eine Inschrift mit dem Namen Ioannes Palaiologos und dem Datum 1443/44 siehe Schneider, Yedikule, 199.

707 Lietzmann, Landmauer, 21, Nr. 12; weitere Literaturangaben bei Meyer-Plath/Schneider, Landmauer, 127, Nr. 11 (Schneider). 
K. 15a/16a: Sechs Quaderlagen. Drei schmale Quaderlagen, die Ziegelband ersetzen. Dreischichtige Ziegelbänder.

T. 16a: Vierseitig. In sehr schlechtem Zustand.

K. 16a/17a: Mauerwerk wie T. 15a/16a.

T. 17a. Halbrund. Quaderwerk.

K. 17a/18a: Nördliche Partie Quaderwerk. Südliche Partie: Quader mit gelegentlichem Ziegeldurchschuß. An den oberen Verbindungsecken mit dem benachbarten Turm (Kasematten) zwölf Ziegelschichten.

T. 18a: Vierseitig. Untere Partie Quaderwerk. Obere Partie unregelmäßige Quader mit Ziegeldurchschuß in den Stoß- und Lagefugen, repariert.

K. 18a/19a: Mauerwerk wie T. 17a/18a.

T. 19a: Vierseitig. Untere Partie Quader mit gelegentlichem Ziegeldurchschuß in den Stoß- und Lagefugen. Obere Partie Quaderwerk (sehr unregelmäßig). An den oberen Verbindungsstellen mit der Kurtine zwölf Ziegelschichten.

K. 19a/20a: Mauerwerk wie T. 17a/18a. Kasematten mit zwölf Ziegelreihen.

T. 20a: Nicht erhalten.

K. 20a/21a: Neuzeitlich restauriert.

T. 21a: Halbrund. Fragmentarisch erhalten.

K. 21a/23a: Vortor von Belgratkapı. Neuzeitlich restauriert.

T. 23a: Halbrund. Nur untere vier Quaderlagen erhalten.

K. 23a/24a: Neuzeitlich restauriert.

T. 24a: Vierseitig. Kurze Flanken. Quaderwerk.

K. 24a/25a: Neuzeitlich renoviert. Fünfschichtige Ziegelbänder fragmentarisch zu erkennen. Ziegelbandhöhe $63 \mathrm{~cm}$, Ziegelbreite $35 \mathrm{~cm}$, Ziegelhöhe 3,5 cm, Mörtelhöhe 6,5 bis $7,5 \mathrm{~cm}$.

T. 25a: Halbrund. Neuzeitlich restauriert.

K. 25a/26a: Neuzeitlich restauriert.

T. 26a: Halbrund. Neuzeitlich restauriert. Fünfschichtige Ziegelbänder fragmentarisch zu erkennen.

K. 26a/27a: Neuzeitlich restauriert.

T. 27a: Vierseitig. Neuzeitlich restauriert. Quaderwerk. Inschrifttafel: Ioannes Palaiologos und Datum 1440 (nicht erhalten). ${ }^{708}$

K. 27a/28a: Neuzeitlich restauriert.

T. 28a: Halbrund. Nur Sockel neuzeitlich wiederhergestellt.

K. 28a/29a: Neuzeitlich restauriert.

708 Lietzmann, Landmauer, 21, Nr. 15; weitere Literaturangaben und Diskussion der Lesung bei Meyer-Plath/Schneider, Landmauer, 127, Nr. 17 (Schneider). 
T. 29a: Vierseitig. War neuzeitlich wiederhergestellt. Eingestürzt (Erdbeben 1999).

K. 29a/30a: Teilweise neuzeitlich restauriert. Fünfschichtige Ziegelbänder zu erkennen (Abb. 118).

T. 30a: Vierseitig. Untere Teilen neuzeitlich wiederhergestellt.

K. 30a/31a: Teilweise neuzeitlich restauriert. Fünfschichtige Ziegelbänder zu erkennen (Abb. 118).

T. 31a: Vierseitig. Vier Quaderlagen. Vier- bzw. fünfschichtige Ziegelbänder. Ziegelbandhöhe $52 \mathrm{~cm}$, Ziegelbreite 33 bis $37 \mathrm{~cm}$, Ziegelhöhe 4,5 cm, Mörtelhöhe 4,5 bis $8 \mathrm{~cm}$, Stoßfuge $2,5 \mathrm{~cm}$.

K. 31a/32a: Neuzeitlich restauriert.

T. 32a: Sechsseitig. Quaderwerk (unregelmäßige Quader).

K. 32a/33a: Neuzeitlich restauriert.

T. 33a: Vierseitig. Erhaltene untere Partien neuzeitlich restauriert.

K. 33a/34a: Neuzeitlich restauriert.

T. 34a: Sechsseitig, sehr hoch und schlank. Neuzeitlich restauriert. Quaderwerk mit unregelmäßigen kleinen Quadern. Inschrifttafel: Ioannes Palaiologos und Datum 1439 (Abb. 59, 60). ${ }^{709}$

K. 34a/36a: Silivrikap1, Vortor. Neuzeitlich restauriert. ${ }^{710}$ Inschrift Leon und Konstantin (nicht erhalten).

T. 36a: Vierseitig. Neuzeitlich restauriert. - An der Westfront P-4.

K. 36a/37a: Neuzeitlich restauriert.

T. 37a: Vierseitig. Nordflanke und größter Teil der Westseite abgestürzt. Quaderwerk. ${ }^{711}$

K. 37a/38a: Fragmentarisch erhalten.

T. 38a: Vierseitig. Quader mit gelegentlichen Ziegeln in den Stoßfugen. Inschrifttafel: Ioannes Palaiologos und Datum 1439 (heute: Skulpturensammlung und Museum für Byzantinische Kunst in Berlin, Inv.-Nr. 6390). ${ }^{712}$

Von T. 38a bis T. 40a: Fragmentarisch erhalten.

T. 40a: Vierseitig. Nur zwei Flanken erhalten. Quader mit gelegentlichen Ziegeln in den Stoß- und Lagefugen.

709 Lietzmann, 22, Nr.16; weitere Literaturangaben und unterschiedliche Lesungen des Datums bei Meyer-Plath/Schneider, 128, Nr. 19 (Schneider).

710 Meyer-Plath/Schneider, Landmauer, 130, Nr.23c (Schneider).

711 Für die nicht mehr erhaltene Inschrifttafel siehe Meyer-Plath/Schneider, Landmauer, 88 (Meyer-Plath).

712 Paspates Meletai, 52, plazierte die Inschrift am Vorturm 37a. Dagegen setzte sie Van Millingen, Walls, 106, an die richtige Stelle, wo heute noch der leere Rahmen zu erkennen ist. Für die Lesung des Datums siehe Wulff, Katalog, 126, Nr. 2220. Lietzmann, Landmauer, 23, Nr. 22 und Meyer-Plath/Schneider, Landmauer, 130, Nr. 25 (Schneider) hielten beide Orte für möglich. - Farbaufnahme bei Asutay-Effenberger, Reparaturinschrift, 69, Nr. 87. 
K. 40a/41a: Fragmentarisch erhalten.

T. 41a: Vierseitig. In schlechtem Zustand. Quaderwerk. Inschrifttafel: Ioannes Palaiologos und Datum 1437 (nicht erhalten) (Abb. 53). ${ }^{713}$

K. 41a/42a: Quaderwerk.

T. 42a: Vierseitig. Nur Reste erhalten. ${ }^{714}$

K. 42a/43a: Untere Partien mit regelmäßigen Quadern und drei- bzw. fünfschichtige Ziegelbänder.

T. 43a: Nicht erhalten.

K. 43a/44a: Wie T. 42a/43a.

T. 44a: Halbrund. Quaderwerk.

K. 44a/45a: Untere Partien mit regelmäßigen Quadern und drei- bzw. fünfschichtige Ziegelbänder.

T. 45a: Vierseitig. Nordflanke nicht erhalten. Quaderwerk.

K. 45a/46a: Fragmentarisch erhalten. Fünfschichtige Ziegelbänder fragmentarisch zu erkennen.

T. 46a: Halbrund. Quader mit zwei bis vier Ziegelschichten in den Lagefugen. An der Verbindungsstelle mit der Kurtine zwölf Ziegelschichten.

K. 46a/47a: Neuzeitlich restauriert.

T. 47a: Vierseitig. Neuzeitlich restauriert.

K. 47a/48a: Neuzeitlich restauriert.

T. 48a: Halbrund. Neuzeitlich restauriert.

K. 48a/49a: Neuzeitlich restauriert.

T. 49a: Vierseitig. Neuzeitlich restauriert.

K. 49a/51a: Mevlevihanekap1, Vortor. Neuzeitlich restauriert. ${ }^{715}$

T. 51a: Vierseitig. Neuzeitlich restauriert.

K. 51a/52a: Neuzeitlich restauriert.

T. 52a: Halbrund. Nur untere Partien erhalten und neuzeitlich restauriert.

713 Paspates, Meletai, 52, plazierte die Inschrift am Turm 41a. Nach Van Millingen, Walls, 106, befand sie sich am Turm. 42a. Lietzmann, Landmauer 23, Nr. 23, und Meyer-Plath/ Schneider, Landmauer, 130, Nr. 27, hielten beide Orte für möglich (Schneider); vgl. 88: „Nach der Mauertechnik muß der Turm um 1439 entstanden sein“ (Meyer-Plath).

714 Meyer-Plath/Schneider, Landmauer, 88, plazieren die in Anm. 715 erwähnte Inschrift hier und datierten den Turm nach dem Mauerwerk um 1439 (Meyer-Plath). Dem Autor zufolge soll es hier den Rest eines Inschrifttafelrahmens gegeben haben, was heute auf Grund der Zerstörung nicht mehr nachvollzogen werden kann. Da aber Meyer-Plath die Inschrifttafel nicht erwähnt, die sich an Turm 41a befindet, ist es möglich, daß hier eine Verwechslung vorliegt.

715 Zum alten Zustand siehe Ousterhout/Başgelen, Postcards, 79, Abb. 47. 
K. 52a/53a: Neuzeitlich restauriert.

T. 53a: Vierseitig. Neuzeitlich restauriert.

K. 53a/54a: Neuzeitlich restauriert.

T. 54a: Halbrund. Quaderlagen. Sechsschichtiges Ziegelband. Quader mit Ziegeln in den Stoß- und Lagefugen. An der nordseitigen Verbindungsstelle mit der Kurtine mehrere Ziegelschichten.

K. 54a/55a: Wiederverwendete Quader und fünfschichtige Ziegelbänder.

T. 55a: Vierseitig. Nur Nordflanke erhalten. Quader und fünfschichtiges Ziegelband bzw. dreischichtiges Quaderband aus Kalkstein, die Ziegelschichten ersetzen. An der Verbindungsstelle mit Kurtine 55a/56a in den oberen Partien zwölf Ziegelschichten.

K. 55a/56a: Untere Partien Quaderwerk. Obere Partien mit unregelmäßigen Quadern mit Ziegeldurchschuß in den Stoß- und Lagefugen (gelegentlich).

T. 56a: Halbrund. Mauerwerk wie T. 55a.

K. 56a/57a: In unteren Teilen regelmäßige Quader und fünfschichtige Ziegelbänder zu erkennen. Obere Teile Quaderwerk.

T. 57a: Halbrund. Mauerwerk wie T. 55a und unregelmäßige Quader mit Ziegeldurchschuß in den Lagefugen.

K. 57a/58a: Mauerwerk wie T. 55a.

T. 58a: Vierseitig. Quader und vier- bzw. fünfschichtige Ziegelbänder. Obere Teile mit unregelmäßigen Quadern mit gelegentlichem Ziegeldurchschuß in der Stoß- und Lagefugen.

K. 58a/60a: Vortor des Romanos-Tors. Nördlichster Abschnitt der Kurtine nicht erhalten. Fünfschichtige Ziegelbänder bzw. vierlagige Kalksteinsteinbänder zwischen den Quadern zu erkennen (Abb. 84). - Die Inschrift in Anm. 699 erwähnt.

T. 60a: Bei Anlage der Millet Caddesi abgebrochen.

K. 60a/61a: Nördliche Teile neuzeitlich renoviert. Südlicher Abschnitt mit unregelmäßigen Quadern mit breiteren Mörtelumrahmungen. Gelegentlich Ziegel in den Stoßfugen.

T. 61a: Vierseitiger Sockel und halbrunder Schaft. Untere Partien erhalten. Neuzeitlich renoviert.

K. 61a/62a: Neuzeitlich restauriert. Ein dreischichtiges Ziegelband zu erkennen.

T. 62a: Halbrund. Quaderwerk und vierschichtiges Kalksteinband. An der Verbindungsstelle mit der Kurtine zwölf Ziegelschichten. Sonst Reparaturen mit unregelmäßigen Quadern und Ziegeln in den Stoßfugen (Abb. 61).

K. 62a/63a: Neuzeitlich restauriert. Sonst wie T. 62a.

T. 63a: Rechteckiger Sockel und polygonaler Schaft. Neuzeitlich restauriert. Quader mit Ziegeldurchschuß in den Stoß- und Lagefugen.

K. 63a/64a: Neuzeitlich restauriert.

T. 64a: Vierseitig. Vier Quaderreihen am Sockel erhalten. Neuzeitlich restauriert. 
K. 64a/66a: Topkapı. Neuzeitlich restauriert.

T. 66a: Vierseitig. Quaderwerk.

K. 66a/67a: Untere Partien erhalten. Unregelmäßige Quader mit zwei- bzw. dreischichtigen Ziegeln in den Lagefugen.

T. 67a: Halbrund. Untere Partien erhalten. Sechs Quaderlagen, dreischichtiges Kalksteinband und dreischichtiges Ziegelband. Ziegelbandhöhe ca. $35 \mathrm{~cm}$, Ziegelbreite $34 \mathrm{~cm}$, Ziegelhöhe 3,5 cm, Mörtelhöhe 4,5 bis 5,5 cm (Abb. 58).

K. 67a/68a: Mauerwerk wie T. 67a. Sonst mit unregelmäßigen Quadern repariert.

T. 68a: Nicht erhalten.

K. 68a/69a: Obere Teile nicht erhalten. Quader mit fünfschichtigen Ziegelbändern. Ziegelbandhöhe $49 \mathrm{~cm}$, Ziegelbreite $34 \mathrm{~cm}$, Ziegelhöhe $3 \mathrm{~cm}$, Mörtelhöhe $4,5 \mathrm{~cm}$.

T. 69a: Vierseitig. Mauerwerk wie K. 68a/69a. Obere Teile mit unregelmäßigen Quadern repariert (Abb. 63, 64).

K. 69a/70a: Mauerwerk wie T. 67a.

T. 70a: Nicht erhalten.

K. 70a/71a: Mauerwerk wie T. 67a.

T.71a: Halbrund. Untere Partien erhalten. Mauerwerk wie T. 67a, aber mit fünfschichtigem Ziegelband. Quader mit fünfschichtigen Ziegelbändern. Ziegelbandhöhe $49 \mathrm{~cm}$, Ziegelbreite $34 \mathrm{~cm}$, Ziegelhöhe 3 bis $5 \mathrm{~cm}$, Mörtelhöhe 3,5 bis $5,5 \mathrm{~cm}$.

K. 71a/72a: Nur ein Teil an der Südseite des Vatan Caddesi erhalten. Mauerwerk wie T. 71a.

T. 72a: Bei Anlage der Vatan Caddesi abgerissen.

K. 72a/73a: Wie T. 72a.

Danach ist die Vormauer fast bis T. 78 a zerstört. ${ }^{716}$

K. 77a/78a: Wenige Partien erhalten. Zum Teil unregelmäßige Quader mit Ziegeldurchschuß. Auch teilweise Ziegelband mit verdeckter Schichttechnik.

T. 78a: Halbrund. Quader und Ziegelbänder mit verdeckter Schichttechnik. Ziegelbandhöhe $51 \mathrm{~cm}$, Ziegelbreite $30 \mathrm{~cm}$, Ziegelhöhe $3 \mathrm{~cm}$, Mörtelhöhe 6 bis $10 \mathrm{~cm}$.

K. 78a/79a: Teilweise erhalten. Nordpartie Quader mit fünfschichtigen Ziegelbändern, auch verdeckte Schichttechnik zu erkennen. Südpartie mit Quadern repariert (Abb. 95).

716 Am Vorturm 77a befanden sich einst zwei Inschriftbänder. Die erste Inschrift erwähnt den Namen Ioannes Palaiologos und das Datum 1432/33. Sie befindet sich heute in den İstanbuler Archäologischen Museen, siehe Meyer-Plath/Schneider, Landmauer, 136, Nr. 45 (Schneider). Die zweite Inschrift war darunter angebracht und erwähnt den Namen Manuel Palaiologos Iagaris, Ratgeber und Finanzberater des Kaisers Konstantin XI. Palaiologos Dragases. (Heute ebenfalls in den İstanbuler Archäologischen Museen); siehe auch Dethier, Bosphor, 55; Van Millingen, Walls, 108; Lietzmann, Landmauer, 26, Nr. 37 und 38. 
T. 79a: Fragmentarisch erhalten. Steht auf Sockel aus länglichen Quadern. Verdeckte Schichttechnik zu erkennen (Abb. 63, 64.

K. 79a/80a: Teilweise erhalten. Mauerwerk wie K. 78a/79a. Sonst Reparaturen mit unregelmäßigen Quadern.

T. 80a: Rechteckig. Acht Quaderlagen und fünfschichtige Ziegelbänder. Ziegelbandhöhe $52 \mathrm{~cm}$, Ziegelbreite $34 \mathrm{~cm}$, Ziegelhöhe 4,5 bis $5 \mathrm{~cm}$, Mörtelhöhe 4,5 bis $5 \mathrm{~cm}$. In sehr schlechtem Zustand. - T. 80a haben Meyer-Plath/Schneider - wie auch T. 80 zweimal angegeben, weshalb ich die Numerierung beibehalte.

K. 80a/80a: Teilweise erhalten. Quader und fünfschichtige Ziegelbänder. Ziegelbandhöhe $52 \mathrm{~cm}$, Ziegelbreite $35 \mathrm{~cm}$, Ziegelhöhe 4,5 bzw. $5 \mathrm{~cm}$, Mörtelhöhe, 5 bzw. $7 \mathrm{~cm}$.

$\mathrm{Ab}$ hier bis Turm 88a ist von der Vormauer fast nichts erhalten.

T. 88a: Halbrund. In sehr schlechtem Zustand. Ziegelbänder sind teilweise zu erkennen. Ziegelbandhöhe $54 \mathrm{~cm}$, Ziegelbreite $35 \mathrm{~cm}$, Ziegelhöhe $4 \mathrm{~cm}$, Mörtelhöhe 3 bis $4 \mathrm{~cm}$ (Abb. 56).

T. 96a: Rechteckig. Neuzeitlich intensiv restauriert. ${ }^{717}$ Sechs große Quaderlagen, fünf bis drei Ziegelschichten. Nach jeder Ziegelschicht werden die Quader kleiner (Abb. 175). An der Stadtseite verdeckte Schichttechnik.

Verbindung zwischen 96a/Tekfur Sarayı: Im unteren Bereichen (stadtseitig) nördlich der Kragkonstruktion verdeckte Schichttechnik. Vier Ziegelbänder mit drei verdeckten Ziegeln. Ziegelbandhöhe $62 \mathrm{~cm}$, Ziegelbreite $33 \mathrm{~cm}$, Ziegelhöhe 4 bis $4,5 \mathrm{~cm}$ (verdeckte Ziegelhöhe 2,5 cm), Mörtelhöhe 9 bis $11 \mathrm{~cm}$ (Abb. 6, 27a, 176, 179).

$$
\text { 4.1.1.3. Graben }{ }^{718}
$$

Graben vor dem Goldenen Tor: Innenmauer Quader mit Ziegeldurchschuß in den Stoß- und Lagefugen. Außenwand fünfschichtige Ziegelbänder. Ziegelbandhöhe $52 \mathrm{~cm}$, Ziegelbreite $35 \mathrm{~cm}$, Ziegelhöhe 3 bis 4,5 cm, Mörtelhöhe 5,5 bis $7 \mathrm{~cm}$. Zum Teil verdeckte Schichttechnik mit Mörtelhöhe $11 \mathrm{~cm}$. Im Mörtel größere Ziegelreste. Ab Eisenbahndamm fast nichts mehr erhalten (Abb. 3, 67).

Ab Turm 15 Richtung Süden besser zu erkennen. Zum Teil verdeckte Schichttechnik (Abb. 66).

Vor Belgratkapı: Neuzeitlich restauriert (Abb. 62, 65).

Vor Silivrikapı: Neuzeitlich restauriert. Wiederverwendete Quader mit fünfschichtigen Ziegelbändern. Ziegelbandhöhe $52 \mathrm{~cm}$, Ziegelbreite $34 \mathrm{~cm}$, Ziegelhöhe 4 bis $4,5 \mathrm{~cm}$, Mörtelhöhe 5,5 bis $7 \mathrm{~cm}$, Stoßfuge $3 \mathrm{~cm}$ (Abb. 12).

Vor Mevlevihanekapi: Neuzeitlich restauriert.

Vor dem Romanos-Tor: Innenwand Quader mit Ziegeldurchschuß in den Stoß- und Lagefugen. Außenwand Schichtenmauerwerk mit fünf- bzw. achtschichtigen Ziegellagen.

717 Für eine alte Aufnahme siehe Ousterhout/Başgelen, Postcards, 91, Abb. 59.

718 Meyer-Plath/Schneider, Landmauer, 92-95 (Meyer-Plath), konnten mehrere Abschnitte noch in besserem Zustand sehen. 
Zwischen Topkapı und Millet Caddesi neuzeitlich und sehr intensiv repariert (Abb. 49).

Der Graben ist nur an der Südseite des Lykos (heute Vatan Caddesi) teilweise erhalten. ${ }^{719}$

\subsubsection{Mumhane-Mauer}

Teilweise erhalten. Drei Quaderlagen $(25 \mathrm{bzw} .40 \mathrm{~cm})$ und fünf- bzw. sechsschichtige Ziegelbänder. Untere Bereiche: Ziegelbandhöhe 63 bis $70 \mathrm{~cm}$, Ziegelbreite 37 bis 40 $\mathrm{cm}$, Ziegelhöhe 5 bis $6,5 \mathrm{~cm}$, Mörtelhöhe 5 bis $7 \mathrm{~cm}$. Obere Bereiche: Fünfschichtige Ziegelbänder. Ziegelbandhöhe 50 bzw. $53 \mathrm{~cm}$, Ziegelbreite 36 bis $38 \mathrm{~cm}$, Ziegelhöhe $4,5 \mathrm{~cm}$, Mörtelhöhe 5 bis $6 \mathrm{~cm}$ (Abb. 22, 136, 137).

\subsubsection{Blachernen-Mauer \\ 4.1.3.1. Komnenen-Mauer (Mauer des Kaisers \\ Manuel I. Komnenos)}

Zusätzlich verwendete Abkürzung: TS = Tekfur Sarayı

K. zwischen TS-Nordturm bis T. 1: Vier unregelmäßige Quaderlagen mit Ziegeldurchschuß in den Lagefugen. Siebenschichtige Ziegelbänder mit ein bzw. zwei Reihen Ziegeldurchschuß. Feldseite: Ziegelbandhöhe $58 \mathrm{~cm}$, Ziegelbreite 29 bis $31 \mathrm{~cm}$, Ziegelhöhe 2 bis $2,5 \mathrm{~cm}$, Mörtelhöhe 5 bis $6 \mathrm{~cm}$. Stadtseite (südlicher Teil): Ziegelbandhöhe $63 \mathrm{~cm}$, Ziegelbreite $30 \mathrm{~cm}$, Ziegelhöhe 2 bis $2,5 \mathrm{~cm}$, Mörtelhöhe 4 bis $5 \mathrm{~cm}$. Stadtseite (nördlicher Teil): Ziegelbandhöhe $56 \mathrm{~cm}$, Ziegelbreite 29 bis $31 \mathrm{~cm}$, Ziegelhöhe 2,5 bis 3,5 cm, Mörtelhöhe 3,5 bis $4 \mathrm{~cm}$ (Abb. 20).

T. 1: Halbrund. Untere zwei Drittel mit unregelmäßigen großen Kalksteinblöcken. Siebenschichtige Ziegelbänder. Ziegelbandhöhe $66 \mathrm{~cm}$, Ziegelbreite $34 \mathrm{~cm}$, Ziegelhöhe 3,5 bis $4 \mathrm{~cm}$, Mörtelhöhe 4 bis $6 \mathrm{~cm}$ (Abb. 19, 147).

K. 1/2: Feldseite siebenschichtige Ziegelbänder. Ziegelbandhöhe $57 \mathrm{~cm}$, Ziegelbreite 29 bis $34 \mathrm{~cm}$, Ziegelhöhe 2,5 bis $4 \mathrm{~cm}$, Mörtelhöhe 3,5 bis $4 \mathrm{~cm}$. Stadtseite mit kleineren Quadern mit Ziegeldurchschuß. Ziegelbandhöhe $63 \mathrm{~cm}$, Ziegelbreite 29 bis $30 \mathrm{~cm}$, Ziegelhöhe 2,5 bis $4 \mathrm{~cm}$, Mörtelhöhe 3,5 bis $4 \mathrm{~cm}$ (Abb. 147).

T. 2: Siebenseitig. Siebenschichtige Ziegelbänder. Ziegelbandhöhe $62 \mathrm{~cm}$, Ziegelbreite $32 \mathrm{~cm}$, Ziegelhöhe 3,5 bis $5 \mathrm{~cm}$, Mörtelhöhe 4 bis $5 \mathrm{~cm}$ (Abb. 147).

K. 2/3: Feldseite siebenschichtige Ziegelbänder. Ziegelbandhöhe $66 \mathrm{~cm}$, Ziegelbreite 31 bis $33 \mathrm{~cm}$, Ziegelhöhe 3 bis 3,5 cm, Mörtelhöhe 5,5 bis 6,5 cm (Abb. 147). Stadtseite mit kleineren Quadern mit Ziegeldurchschuß. Östlich der Poterne: neuenschichtige Ziegellagen. Ziegelbandhöhe 63 bzw. $66 \mathrm{~cm}$, Ziegelhöhe 2,5 bzw. 3,5 und $4 \mathrm{~cm}$, Ziegelbreite 29 bis $30 \mathrm{~cm}$, Mörtelhöhe $4 \mathrm{~cm}$ (Abb. 153, 161).

T. 3: Halbrund. Siebenschichtige Ziegelbänder. Feldseite Ziegelbandhöhe $62 \mathrm{~cm}$, Ziegelbreite $30 \mathrm{~cm}$, Ziegelhöhe 3 bis $3,5 \mathrm{~cm}$, Mörtelhöhe 4 bis $6 \mathrm{~cm}$. Obere Partien mit

719 Für eine alte Aufnahme des Zustands zu Beginn des 20. Jahrhunderts siehe Ousterhout/ Başgelen, Postcards, 74, Abb. 42 und 75, Abb. 43. 
kleineren unregelmäßigen Quadern repariert (Abb. 147). Auf einem Quader reliefiertes Kreuz mit geschweiften Enden.

K. 3/4: Feldseite neunschichtige Ziegelbänder. Ziegelbandhöhe $67 \mathrm{~cm}$, Ziegelbreite 30 bis $35 \mathrm{~cm}$, Ziegelhöhe 3,5 bis $4 \mathrm{~cm}$, Mörtelhöhe 4 bis $6 \mathrm{~cm}$. Stadtseite mit kleineren Quadern und achtschichtige Ziegelbänder. Ziegelbandhöhe $62 \mathrm{~cm}$, Ziegelhöhe $2,5 \mathrm{~cm}$ bis $4 \mathrm{~cm}$, Ziegelbreite 28 bis $30 \mathrm{~cm}$, Mörtelhöhe 4 bis $6 \mathrm{~cm}$. Kalksteinblöcke $50 \times 50$ bzw. 70 x $41 \mathrm{~cm}$.

T. 4: Achtseitig, Siebenschichtige Ziegelbänder. Ziegelbandhöhe $66 \mathrm{~cm}$, Ziegelbreite ca. $35 \mathrm{~cm}$, Ziegelhöhe 3 bis $4,5 \mathrm{~cm}$, Mörtelhöhe 5,5 bis $6,5 \mathrm{~cm}$. Obere Teile mit unregelmäßigen Quadern repariert.

K. 4/5: Wie K. 3/4

T. 5: Hufeisenförmig. Sieben- bis neunschichtige Ziegelbandhöhe $70 \mathrm{~cm}$, Ziegelbreite 30 bis $37 \mathrm{~cm}$, Ziegelhöhe 3,5 bis $5 \mathrm{~cm}$, Mörtelhöhe 5,5 bis $7 \mathrm{~cm}$. Obere Teile mit kleineren unregelmäßigen Quadern repariert (Abb. 159). Im Turm sechs- bis neunschichtige Ziegelbänder, Ziegelbandhöhe $51 \mathrm{~cm}$, Ziegelhöhe $2,5 \mathrm{~cm}$, Ziegelbreite 28 bis $30 \mathrm{~cm}$, Mörtelhöhe $5 \mathrm{~cm}$.

K. 5/6: Feldseite Ziegelbandhöhe $69 \mathrm{~cm}$, Ziegelbreite 30 bis $37 \mathrm{~cm}$, Ziegelhöhe $4 \mathrm{~cm}$, Mörtelhöhe 6 bis $7 \mathrm{~cm}$ (Abb. 149). - V-6. Stadtseite kleinere Quader, neunschichtige Ziegelbänder. Ziegelbandhöhe $72 \mathrm{~cm}$, Ziegelbreite 28 bis $30 \mathrm{~cm}$, Ziegelhöhe 3 bis $3,5 \mathrm{~cm}$, Mörtelhöhe $4 \mathrm{~cm}$.

T. 6: Neunseitig. Achtschichtige Ziegelbänder. Ziegelbandhöhe $73 \mathrm{~cm}$, Ziegelbreite 30 bis $40 \mathrm{~cm}$, Ziegelhöhe 3 bis $5 \mathrm{~cm}$, Mörtelhöhe $6 \mathrm{~cm}$. Obere Teile mit unregelmäßigen Quadern repariert. - KO-6.

K. 6/7: Kurtine wird durch Eğrikapı unterbrochen (Abb. 156, 157). Achtschichtige Ziegelbänder und Quader mit Ziegeldurchschuß in den Lagefugen. Ziegelbandhöhe $85 \mathrm{~cm}$, Ziegelbreite $31 \mathrm{~cm}$, Ziegelhöhe 3,5 cm, Mörtelhöhe 6 bis $7 \mathrm{~cm}$. An der Stadtseite in den unteren Bereichen Ziegelbänder mit verdeckter Schichttechnik.

T. 7: Achtseitig. Neunschichtige Ziegelbänder. Ziegelbandhöhe $69 \mathrm{~cm}$, Ziegelbreite 30 bis $37 \mathrm{~cm}$, Ziegelhöhe 3 bis $5 \mathrm{~cm}$, Mörtelhöhe 4 bis $5 \mathrm{~cm}$ (Abb. 156).

K. 7/8: Feldseite Ziegelbandhöhe $64 \mathrm{~cm}$, Ziegelbreite 30 bis $35 \mathrm{~cm}$, Ziegelhöhe 3 bis $4 \mathrm{~cm}$, Mörtelhöhe $6 \mathrm{~cm}$. Stadtseite wie K. 6/7.

T. 8: Achtseitig. Neuenschichtige Ziegelbänder. Ziegelbandhöhe $78 \mathrm{~cm}$, Ziegelbreite 34 bis $37 \mathrm{~cm}$, Ziegelhöhe 3,5 bis $5 \mathrm{~cm}$, Mörtelhöhe 4 bis $5 \mathrm{~cm}$. An beiden Seiten des Turmeingangs Reste einer späteren Treppe. Fünfschichtige Ziegelbänder, Quader mit ein bis drei Reihen Ziegeldurchschuß. Ziegelbandhöhe $63 \mathrm{~cm}$, Ziegelbreite 28 bis $30 \mathrm{~cm}$, Ziegelhöhe 2 bis 2,5 cm, Mörtelhöhe 3 bis $5 \mathrm{~cm}$ (Abb. 155).

K. 8/9: Feldseite siebenschichtige Ziegelbänder. Ziegelbandhöhe $80 \mathrm{~cm}$, Ziegelbreite 35 bis $37 \mathrm{~cm}$, Ziegelhöhe $5 \mathrm{~cm}$, Mörtelhöhe $6 \mathrm{~cm}$. Stadtseite Ziegelbandhöhe 68 bis $70 \mathrm{~cm}$, Ziegelbreite 28 bis $35 \mathrm{~cm}$, Ziegelhöhe 2,5 bis $4 \mathrm{~cm}$, Mörtelhöhe 3,5 bis $5 \mathrm{~cm}$. Zum Teil verdeckte Schichttechnik mit $11 \mathrm{~cm}$ Mörtelhöhe (Abb. 192). - P-9.

T. 9: Rechteckig. Ziegelbandhöhe $78 \mathrm{~cm}$, Ziegelbreite $35 \mathrm{~cm}$, Ziegelhöhe $6 \mathrm{~cm}$, Mörtelhöhe $5 \mathrm{~cm}$. Ziegeldurchschuß in den Lagefugen. Zum Teil verdeckte Schichttechnik (Abb. 150). 
K. 9/10: Nicht im Verband mit T. 9 (Abb. 21). Sieben- bis neuenschichtige Ziegelbänder, wiederverwendete Quader mit ein- bzw. zweischichtigen Ziegeln in den Lagefugen. Feldseite Ziegelbandhöhe $75 \mathrm{~cm}$, Ziegelbreite 25 bis $30 \mathrm{~cm}$, Ziegelhöhe $2,5 \mathrm{~cm}$, Mörtelhöhe $7 \mathrm{~cm}$ (Abb. 150). Stadtseite Ziegelbandhöhe $63 \mathrm{~cm}$, Ziegelbreite $30 \mathrm{~cm}$, Ziegelhöhe 2,5, 3 bzw. 3,5 cm, Mörtelhöhe $4 \mathrm{~cm}$.

T. 10: In sehr schlechtem Zustand. Ähnlich wie K. 9/10. - K-15 an der Südwestecke des Turms.

K. 10/11: Wie T. 10.

T. 11: Rechteckig. Innen und außen neunschichtige Ziegelbänder. Ziegelbandhöhe $63 \mathrm{~cm}$, Ziegelbreite $30 \mathrm{~cm}$, Ziegelhöhe 3 bis $4 \mathrm{~cm}$, Mörtelhöhe 3,5 bis $5 \mathrm{~cm}$. Auch Ziegelband mit verdeckter Schichttechnik, Mörtelhöhe: 11 bis $12 \mathrm{~cm}$. Obere Teile mit Quadern repariert (Abb. 148, 152).

K. 11/12: Wie T. 11. An der Stadtseite nördlich des Turms eine Nische mit dreifach abgestuftem Bogen, Zickzackmuster und verdeckter Schichttechnik (Abb. 148). - KP-1, KP-2, KP-3 (Abb. 159a, b, c).

T. 12: Rechteckig. Mauerwerk wie T. 11 (Abb. 148). Ziegelbandhöhe 75 bis $80 \mathrm{~cm}$. Obere Teile mit Quadern repariert. Die Nische an der oberen Etage mit polychromen Bögen (Abb. 24, 154).

\subsubsection{Von der Komnenen-Mauer bis zum Goldenen Horn}

K. 12/13: Quaderwerk (Abb. 160). Inschrifttafel: Ioannes Palaiologos und Datum 1441 (nicht erhalten). An der Stadtseite nahe T. 12 wiederverwendetes Inschriftstück: Datum 1317 bzw. 1318 (nicht erhalten). ${ }^{720}$ Die stadtseitigen Nischen mit polychromen Bögen (Abb. 162). - K-16, K-19.

T. 13: Vierseitig. Nordflanke und obere Partien abgestürzt (Abb. 168). Wiederverwendete unregelmäßige fünf- bzw. siebenlagige Quader und fünf- bis sechsschichtige Ziegelbänder. Ziegelbandhöhe ca. $38 \mathrm{~cm}$, Ziegelbreite 15 bis $30 \mathrm{~cm}$, Ziegelhöhe 2,5 bis $5 \mathrm{~cm}$, Mörtelhöhe $3 \mathrm{~cm}$ (Abb. 166). An der Westseite Inschrift: Isaakios II. Angelos (Abb. 167). ${ }^{721}$

K. 13 bis „Vorsprung“ (K. 13/14): Cloissonnémauerwerk mit verdeckter Schichttechnik. Ziegelbandhöhe $65 \mathrm{~cm}$, Ziegelbreite $33-35 \mathrm{~cm}$, Ziegelhöhe $4 \mathrm{~cm}$, Mörtelhöhe 11 bis $12 \mathrm{~cm}$ (Abb. 23, 164). Ähnliches Mauerwerk kommt auch (in sehr schlechtem Zustand) an der nach Osten umbiegenden Mauer an der Stadtseite vor (Abb. 26, 162, 163).

„Vorsprung“: Obere Partien Quaderwerk. Untere Partien Ziegelbänder mit verdeckter Schichttechnik. Ziegelbandhöhe $75 \mathrm{~cm}$, Ziegelbreite $33 \mathrm{~cm}$, Ziegelhöhe $4 \mathrm{~cm}$, Mörtelhöhe 11 bis $12 \mathrm{~cm}$ (Abb. 23, 174).

T. 14 (sog. Isaakios Angelos-Turm): Neuzeitlich restauriert. Drei bzw. fünf Quaderlagen (groß). Fünfschichtige Ziegelbänder (Abb. 25, 170). - Zahlreiche Spolien, darunter I-9.

720 Vgl. Anm. 552 und 568.

721 Vgl. Anm. 579. 
Nördlicher Anbau: Kleine Quader mit Ziegeldurchschuß in den Lagefugen sowie fünfschichtige Ziegelbänder (52 cm hoch) (Abb. 25, 170).

Substruktionsfassade: Vier- bis fünflagige Quader. Ziegelband mit verdeckter Schichttechnik (Abb. 25, 169, 171).

14-Meter-Mauer: Feldseite neuzeitlich restauriert. In unteren Partien große Blöcke. Fünfschichtige Ziegelbänder. Ziegelbandhöhe $58 \mathrm{~cm}$, Ziegelbreite 33 bis $37 \mathrm{~cm}$, Ziegelhöhe 4 bis $5 \mathrm{~cm}$, Mörtelhöhe 5 bis $7 \mathrm{~cm}$ (Abb. 138, 140, 169). - V-3. Stadtseite Ziegelbandhöhe $52 \mathrm{~cm}$, Ziegelbreite 32 bis $37 \mathrm{~cm}$, Ziegelhöhe 2,5 bis $3,5 \mathrm{~cm}$, Mörtelhöhe 4 bis $6 \mathrm{~cm}$ (Abb. 141).

Nördliche Begrenzung der hinteren Substruktion: Mehrfach repariert, in schlechtem Zustand. Ziegelbreite $47 \mathrm{~cm}$, Ziegelhöhe 4 bis $6 \mathrm{~cm}$, Mörtelhöhe $6 \mathrm{~cm}$ (Abb. 165).

Leon-Vorwerk: Neuzeitlich restauriert. Ca. 2 m unter dem Schutt (Abb. 142).

Südliche Bastion: Länglich geschnittene Kalksteinblöcke und fünfschichtige Ziegel. Ziegelbandhöhe $55 \mathrm{~cm}$, Ziegelbreite $38 \mathrm{~cm}$, Ziegelhöhe $5 \mathrm{~cm}$, Mörtelhöhe 5 bis $6 \mathrm{~cm}$ (Abb. 138). Reparaturinschrift: Michael und Theophilos. Inschriftblock mit Jahresangabe $821 / 22 .^{72}$

Im Bereich des zugemauerten Tors ebenfalls neuzeitlich intensiv renoviert. Sieben bis neun Quaderlagen und fünfschichtige Ziegelbänder sind zu erkennen.

Südlich des zugemauerten Tors: Ziegelbandhöhe $62 \mathrm{~cm}$, Ziegelbreite 35 bis $40 \mathrm{~cm}$, Ziegelhöhe 4 bis $5 \mathrm{~cm}$, Mörtelhöhe $6 \mathrm{~cm}$.

Nördlich des zugemauerten Tors: Ziegelbandhöhe $46 \mathrm{~cm}$, Ziegelbreite 33 bis $38 \mathrm{~cm}$, Ziegelhöhe 3,5 bis $4 \mathrm{~cm}$, Mörtelhöhe $5 \mathrm{~cm}$.

T. 15-16-18 vor dem sog. Pteron (Abb. 25): Neuzeitlich restauriert. Mauerwerk ähnlich T. 88 der Landmauer. An T. 15 Inschriftrest: Theophilos. ${ }^{723}$ An T. 16 Quader mit reliefiertem Senmurv. ${ }^{724}$ Werte (von der Rückseite des T. 15 aufgenommen): Ziegelbandhöhe $47 \mathrm{~cm}$, Ziegelbreite 30 bis $35 \mathrm{~cm}$, Ziegelhöhe 2,5 bis $5 \mathrm{~cm}$, Mörtelhöhe 4 bis $6 \mathrm{~cm}$. (Abb. 143, Turm 15).

K 15/16: Feldseite intensiv restauriert. Fünfschichtige Ziegelbänder erkennbar. Ziegelbandhöhe $54 \mathrm{~cm}$, Ziegelbreite 31 bis $37 \mathrm{~cm}$, Ziegelhöhe 3,5 bis $5 \mathrm{~cm}$, Mörtelhöhe 4 bis $6 \mathrm{~cm}$. Stadtseite in schlechtem Zustand. Ziegelbreite $32 \mathrm{~cm}$, Ziegelhöhe 2,5 bis 3,5 cm, Mörtelhöhe $5 \mathrm{~cm}$. Zugemauerte Nische Ziegelbreite $32 \mathrm{~cm}$, Ziegelhöhe $5 \mathrm{~cm}$, Mörtelhöhe $7 \mathrm{~cm}$.

Zweifronten-Wehrgang (sog. Pteron): Neuzeitlich restauriert. Ziegelbandhöhe 47 bzw. $54 \mathrm{~cm}$, Ziegelbreite 32 bis $38 \mathrm{~cm}$, Ziegelhöhe 3 bis $4 \mathrm{~cm}$, Mörtelhöhe 5 bis $6 \mathrm{~cm}$ (Abb. 144).

Unterhalb des Treppenbogens: Ziegelbreite 35 bis $38 \mathrm{~cm}$, Ziegelhöhe 4 bis $5 \mathrm{~cm}$, Mörtelhöhe 5,5 bis $6 \mathrm{~cm}$.

Stadtseitige Treppe: fünfschichtige Ziegelbänder. Ziegelbandhöhe $62 \mathrm{~cm}$, Ziegelbreite $35 \mathrm{~cm}$, Ziegelhöhe $5 \mathrm{~cm}$, Mörtelhöhe 6 bis $9 \mathrm{~cm}$ (Abb. 145).

722 Meyer-Plath/Schneider, Landmauer, 141, Nr.64a und 64b (Schneider).

723 Meyer-Plath/Schneider, Landmauer, 141, Nr. 62 (Schneider).

724 Eyice, Senmurw, 110-119, Taf. 27a-c, 28a. 
T. 19: Sog. Nikolaus-Turm. Sehr unregelmäßige fünflagige Quader mit Ziegeldurchschuß in den Lagefugen (zum Teil in den Stoßfugen). Fünfschichtige Ziegelbänder zum Teil aus sehr dünnen Ziegeln. Ziegelbandhöhe $62 \mathrm{~cm}$, Ziegelbreite $45 \mathrm{~cm}$, Ziegelhöhe 4 bis $5 \mathrm{~cm}$, Mörtelhöhe $6 \mathrm{~cm}$. Reparaturinschrift: Romanos. ${ }^{725}$

\subsection{Besondere Merkmale des Mauerwerks}

Die Merkmale des Mauerwerks wurden in den vorangegangenen Ausführungen auch unter Heranziehung der Sekundärliteratur im einzelnen dargelegt. Im folgenden soll ein zusammenfassender Überblick gegeben werden.

Außer in den Inschriften am Vortor des Mevlevihanekapı (Abb. 42, 79) ${ }^{726}$ ist Kaiser Theodosius II. nirgendwo an der Mauer namentlich erwähnt. Dennoch können einige Abschnitte auf Grund bestimmter Merkmale in die Entstehungszeit datiert werden. Die erste auffallende Besonderheit des theodosianischen Mauerwerks der Hauptmauer - abgesehen vom polychromen Sichtmauerwerk bzw. Schichtenmauerwerk - sind regelmäßige, scharfkantige und länglich geschnittene Quader, die mit geringen Fugen sorgfältig verlegt sind. Die Zahl der Quaderlagen unterscheidet sich von Turm zu Turm. Die fünfschichtigen Ziegelbänder sind in der Regel $45 \mathrm{~cm}$ hoch (mit Grenzwerten 34 bzw. $59 \mathrm{~cm}$ ). Die Ziegel sind 37 bzw. $38-40 \mathrm{~cm}$ breit und 4,5 bis $5 \mathrm{~cm}$ hoch. Die Höhe des rötlichen Mörtels (mit Ziegelbrocken) stimmt mit der Ziegelhöhe überein. Theodosianisches Mauerwerk ist an der Hauptmauer besonders im Bereich K. 8/9 (Abb. 127), K. 13/14, T. 14, K. 14/15, T. 16, T. 17 (Abb. 4), T. 71, K. 71/72, an der Wehrgangstreppe hinter Sulukulekapı (Abb. 94) und an den unteren Partien mehrerer Türme und Kurtinen festzustellen. Das Mauerwerk der von Meyer-Plath/Schneider der theodosianischen Zeit zugeschrieben Teile der Vormauer unterscheidet sich von der Hauptmauer dadurch, daß die Quader hier nicht regelmäßig geschnitten sind und breitere Fugen aufweisen. Als ein Merkmal der Vormauer treten besonders in den unteren Partien drei- bis fünflagige Kalksteinbänder und weniger als fünf Ziegelschichten auf (Abb. 58, 61). ${ }^{727}$ Mehrere dieser Türme wurden später in einer anderen Mauertechnik repariert.

Abgesehen von einer Inschrift, die am Torsturz des Mevlevihanekapı angebracht wurde und Kaiser Justin II. (565-578) sowie seiner Frau Sophia erwähnt (Abb. 42, 79) ${ }^{728}$ ist kein weiterer Mauerabschnitt inschriftlich in das 6. Jahrhundert zu datieren. Die Mumhane-Mauer, die m.E. bald nach 626

725 Meyer-Plath/Schneider, Landmauer, 141, Nr.65 (Schneider), identifizierten ihn mit Kaiser Romanos III. Argyros (1028-1034).

726 Vgl. Anm. 124.

727 Meyer-Plath/Schneider, Landmauer, 24.

728 Meyer-Plath/Schneider, Landmauer, 133, Nr. 34 (Schneider); Ševčenko, Inscription, 18. 
datiert werden kann (siehe Abschnitt 2.2.1.), unterscheidet sich von den Mauerteilen aus theodosianischer Zeit in erster Linie dadurch, daß hier neben fünfschichtigen auch sechsschichtige Ziegelbänder vorkommen und das Mörtelbett heller und erheblich höher ist (Abb. 22, 137). Turm 40 (Abb.77), der Nordturm des sog. 3. Nebentors, wird wegen der auf einem Marmorblock angebrachten Inschrift mit Monogramm in das Jahr 685 datiert (Abb. 199). ${ }^{729}$ Die acht- bzw. mehrlagigen und sorgfältig geschnittenen Quader werden von fünfschichtigen Ziegelbändern ( $49 \mathrm{~cm}$ hoch) unterbrochen. Sowohl die Fugenhöhe zwischen den Quadern als auch die Mörtelhöhe der Ziegelbänder sind deutlich zu erkennen. ${ }^{730} \mathrm{Da}$ die Ziegel des 7 . Jahrhunderts gegenüber den theodosianischen Ziegeln kleiner geworden sind, kann hier eine Wiederverwendung von theodosianischem Material angenommen werden. ${ }^{731}$ Wegen der Ähnlichkeit des Mauerwerks sowie der auf drei Blöcken angebrachten Monogramme wird die obere Partie von Turm 46 ebenfalls dem 7. Jahrhundert zugeschrieben. ${ }^{732}$ Die Reparaturinschriften an den Türmen 18 (Abb. 50), 19 (Abb. 54), 25 (Abb. 52), 34, 37, 45 (Abb. 51), 47, 48, 53, 55 und 56 erwähnen Leon und Konstantin. Mehrere davon sind auf Kaiser Leon III. (717-741) und Konstantin V. (741-775) zu beziehen, die unmittelbar nach dem großen Erdbeben von 740 die Mauer wiederhergestellt haben. ${ }^{733}$ Die Inschrift an Turm 54 erwähnt zwar keinen Kaiser, gehört aber wohl zur gleichen Serie (Abb. 5). Dazu kann auch Turm 63 gerechnet werden (Abb. 49). Steinlagen unterscheiden sich auch in dieser Zeit von Turm zu Turm, und die Ziegelbandhöhe, soweit aufgenommen werden konnte, schwankt zwischen ca. 48 und $52 \mathrm{~cm}$. Man erkennt an allen diesen Türmen das Bemühen, sich dem theodosianischen Vorbild anzugleichen. Eine Betrachtung der Mauern aus der Entfernung zeigt, daß dies in gewissen Maßen auch erreicht wurde (Abb. 49). Doch das wiederverwendete

729 Vgl. Anm. 118.

730 Meyer-Plath/Schneider, Landmauer, 24 (Meyer-Plath); Foss, Fortifications, 53.

731 Kâhya, Tuğla, 171-183; vgl. Mitchell/Aran/Lieggett, Construction, 467-487. - Für eine Diskussion über die Reduktion der Ziegeldimensionen nach der justinianischen Zeit siehe Bardill, Brickstamps, 102-106.

732 Vgl. Anm.689.

733 Theophanes, Chronographia ad. ann.739/40, 412/6-21, hier 9-10; Mango, Chronicle, 572; Schreiner, Kleinchroniken I, 44, Nr. 1, \$15; II, 85; III, 14, \$15 (Übersetzung). Zum Erdbeben von 740 (Downey, Earthquakes, 598; Grumel, Chronologie, 479; Guidoboni, Catalogue, 364-365, Nr.247, mit weiteren Quellen) siehe auch Rochow, Theophanes, 136. - Cameron/Herrin, Parastaseis, 20, zufolge stammen die Reparaturen aus dem Jahre 718; siehe dagegen Kresten, Leon III., 21 -52. Foss, Fortifications, $53-$ 54, setzt die Türme 45, 47, 48, 55, 56 in den Zeitraum 775/780, wobei hier ähnliches Mauerwerk wie an den Türmen, die 740/41 datiert werden, zu erkennen ist; vgl. MeyerPlath/Schneider, Landmauer, 76-77, 154-155 (Meyer-Plath). 
Material und die dadurch bedingten breiteren Stoß- Lagefugen sowie die $35 \mathrm{~cm}$ breiten Ziegel lassen den Zeitunterschied deutlich werden. ${ }^{734}$

Trotz intensiver Reparaturen sind noch Teile des ursprünglichen Mauerwerks des Leon-Vorwerks (um 813) zu erkennen (Abb. 142). Dazu kann man vor allem die südliche Partie des zugemauerten Tors (sog. Blachernen-Tor) rechnen, wo südlich die Bastion von Michael und Theophilos anschließt (Abb. 35, 138). Auffällig sind hier die wiederverwendeten kleineren Quader und die über $60 \mathrm{~cm}$ Höhe erreichenden fünfschichtigen Ziegelbänder. Die Ziegel sind ebenfalls wiederverwendet. Ihre Breite variiert zwischen 35 und $40 \mathrm{~cm}$, ihre Höhe beträgt 4 bis $5 \mathrm{~cm}$, der rötliche Mörtel (es sollen hier die späteren Reparaturen immer beachtet werden) ist $6 \mathrm{bzw}$. über $6 \mathrm{~cm}$ hoch. Ähnliches Mauerwerk begegnet am Vortor des Goldenen Tors (Abb. 7, 8) (siehe Abschnitt 2.1.5.), an der stadtseitigen Kurtine 82/83 der Landmauer (Abb. 106) und an der Rückseite von Turm 86, dem südlichen Torturm von Edirnekap1 (Abb. 98). Auch in den unteren Partien von Turm 19 (sog. Nikolaos-Turm) an der Nordwestecke des Leon-Vorwerks begegnen Ziegelbänder von über $60 \mathrm{~cm}$ Höhe, wo aber auch ganz unterschiedliche Ziegeldimensionen vorkommen. ${ }^{735}$ An der südlichen Bastion des von Michael und Theophilos erweiterten Vorwerks kommen scharfkantig geschnittene Quader und fünfschichtige Ziegelbänder vor (Abb. 138), wobei die Zahl der Steinlagen reduziert ist. Die drei Türme des Kaisers Theophilos (829-842) am Zweifronten-Wehrgang (Abb. 35, 143) unterscheiden sich von der erwähnten südlichen Bastion des Leon-Vorwerks (Abb. 138). Hier wurden in den unteren Teilen größere Blöcke eingesetzt, die von sechs- bzw. siebenschichtigen Ziegelbändern unterbrochen werden. Die oberen Partien sind gänzlich aus Ziegel errichtet. Das Mauerwerk zwischen den feldseitigen Kurtinen 84/85 (Abb. 111), 85/86 (Abb. 103), 87/88 (Abb. 102), Turm 88 (Abb. 56) und Kurtine 88/89 (Abb. 56) von Edirnekap1 läßt sich mit den inschriftlich Kaiser Theophilos zugeschriebenen Türmen am Zweifronten-Wehrgang vergleichen (Abb. 25, 143). ${ }^{736}$ Eine im Bereich von Edirnekap1 einst befindliche Inschrift mit der Jahreszahl 826/27 (Michael II.) bestätigt, wie bereits oben erwähnt (Abschnitt 1.2.2.), daß diese Mauerpartien kurz vor der Regierungszeit des Kaisers Theophilos repariert wurden.

Die Inschrift an Turm 1 der Theodosianischen Landmauer erwähnt den Namen eines Kaisers Basileios. ${ }^{737}$ Während im untersten Teil des Turms große Blöcke benutzt wurden, sind die aufgehenden Partien aus Schichtenmauerwerk

734 Diese Ziegelbreite entspricht den Ziegeln der im gleichen Zeitraum reparierten Teile der Eirenen-Kirche; siehe dazu Peschlow, Irenenkirche, Liste nach 224.

735 Der obere Abschnitt des Turms trägt eine Reparaturinschrift mit dem Kaisernamen Romanos, vgl. Anm.725.

736 Vgl. Foss, Fortifications, 55, wobei Turm 88 mit Turm 86 verwechselt wurde und von einer Strecke von Turm 81 bis 89 die Rede ist.

737 Vgl. Anm. 663. 
mit kleineren Quadern errichtet (Abb. 112). Ansonsten zeigt das gesamte Mauerwerk eine gewisse Ähnlichkeit mit dem der 740/41 reparierten Türme. ${ }^{738}$

Turm 57 besitzt eine Ziegelinschrift mit dem Namen des Kaisers Konstantin Porphyrogennetos, die entweder mit Konstantin VI. (780-797) oder mit Konstantin VII. (913-959) verbunden werden kann. ${ }^{739}$ Die kleinen und länglich geschnittenen, mehrlagigen Quader sind auf sorgfältige Weise wiederverwendet worden. Die Ziegelbreite beträgt $34 \mathrm{~cm}$, hohe Mörtelbetten sind zum Teil erkennbar. Turm 4 (Abb. 1) weist eine Inschrift auf, die den Namen des Kaisers Romanos wiedergibt. ${ }^{740}$ Sowohl die Höhe der Mörtelfugen (bis $8 \mathrm{~cm}$ ) als auch die Ziegelmaße von $34 \mathrm{~cm},{ }^{741}$ ebenso die kleinen Quader lassen den Turm mit Turm 57 vergleichen. Die Ähnlichkeit mit den in das 10. Jahrhundert datierbaren Bereichen am Vortors des Goldenen Tors ist augenscheinlich (Abb. 7, 8) (siehe Abschnitt 2.1.5.). Das sehr hohe Mörtelbett, das gelegentlich bis zu 7 bzw. $8 \mathrm{~cm}$ erreicht, kommt auch an den Türmen 3, 5, 14, 29, 32, 39, 42, 73, 74, 75 (Abb. 55), 83 (Abb. 109) und 84 (Abb. 111) vor; sie können ebenfalls in das 10. Jahrhundert datiert werden.

Der erste auffallende Unterschied zwischen dem Mauerwerk des 12. Jahrhunderts und demjenigen aus den vorherigen Epochen ist die Verwendung der verdeckten Schichttechnik und noch kleinerer Ziegelformate, wie z. B. an den Blachernen-Substruktionen (Abb. 169, 171) oder an verschiedenen Stellen der Komnenen-Mauer (Abb. 151, 152, 158). Um diese Zeit schwankt die Ziegelbreite in der Regel zwischen 30 und 34 bzw. $35 \mathrm{~cm}$. In einigen Abschnitten kommen noch kleinere Ziegel vor ${ }^{742}$ wobei aber auch eine Ziegelbreite von 37 bzw. 38 begegnet. Am Südtrakt der Komnenen-Mauer wurden überwiegend größere Quader verwendet (Abb. 149, 150). Das an der Südseite des „Vorsprungs" der Blachernen-Mauer in Verbindung mit verdeckter Schichttechnik vorkommende Cloissonnémauerwerk (Abb. 23, 26) ist ebenfalls in die komnenische Epoche, jedoch vor der Komnenen-Mauer zu datieren (siehe Abschnitt 3.3.). ${ }^{743}$ Auch an der Landmauer ist verdeckte Schichttechnik vor allem an der Vormauer wie an Vorturm 79a (Abb. 63, 64) und den benachbarten Kurtinenresten sowie am Graben (Abb. 67) zu beobachten.

738 Eine weitere Inschrift mit dem Namen Basileios befand sich am Nordturm von Silivrikapı. Der Turm ist neuzeitlich überrepariert. In den unteren Teilen größere Blöcke. Ziegelbandhöhe $52 \mathrm{~cm}$, Ziegelbreite $37 \mathrm{~cm}$, Ziegelhöhe 4,5 bis $5,5 \mathrm{~cm}$, Mörtelhöhe 4,5 bis $5 \mathrm{~cm}$.

739 Vgl. Anm. 696.

740 Vgl. Anm. 669.

741 Auch in der Myrelaion-Kirche (Anfang 10. Jahrhundert) schwanken die Ziegelbreiten zwischen 30 und $35 \mathrm{~cm}$; vgl. Kâhya, Tuğla, 172 (Liste).

742 In der Pantokrator-Kirche (12. Jahrhundert) schwankt die Ziegelbreite zwischen 33,5 und $39 \mathrm{~cm}$; vgl. Kahya, Tuğla, 172 (Liste).

743 Foss, Fortifications, 76, datiert das Mauerwerk um 1170. 
Es gibt keinerlei Indizien, um einen bestimmten Mauerabschnitt der Lateinerzeit zuschreiben zu können. Dagegen befinden sich an der gesamten Mauer mehrere Bereiche, die in palaiologischer Zeit repariert oder von Grund auf neu errichtet wurden, wobei man altes Baumaterial intensiv wiederbenutzt hat. ${ }^{744}$ Aus der frühpalaiologischen Epoche ist keine Inschrift in situ erhalten. Eine Maueranalyse der reparierten Teile der Blachernen-Mauer in Verbindung mit der hier einst befindlichen Inschrift Andronikos' II. Palaiologos, die in den beiden Torverzeichnissen registriert wurde (siehe Abschnitt 3.2.), ${ }^{745}$ läßt erkennen, daß frühpalaiologisches Mauerwerk auf den ersten Blick dem komnenischen sehr ähnlich ist. Hier tritt aber die verdeckte Schichttechnik nicht mehr in Erscheinung, und die Ziegel sind überwiegend sehr dünn. Das ist besonders an Reparaturstellen des Südtrakts der Komnenen-Mauer nachzuvollziehen (Abb. 20, 155). Das unregelmäßige Mauerwerk an Turm 13 kann ebenfalls dieser Zeit zugeschrieben werden (Abb. 166-168). Quader und Ziegel scheinen dort zum wiederholten Male wiederverwendet worden zu sein.

In die palaiologische Zeit sind auch diejenigen Mauerabschnitte zu datieren, die aus Quadern mit ein bis zwei Ziegelreihen in den Lagefugen errichtet wurden. An den Reparaturstellen in den oberen Teilen von Kurtine 85/86 (Abb. 103), die mit diesem Mauerwerk ausgeführt sind, werden auf Grund eines in Abschriften überlieferten Gedichts, das Kaiser Alexios III. Angelos und das Charisios-Tor erwähnt, in die Zeit um 1197 datiert. ${ }^{746}$ Wie oben gezeigt wurde, handelt es sich beim Charisios-Tor nicht um Edirnekapı, sondern um Sulukulekapı (siehe Abschnitt 2.2.4.3.), weshalb die Reparaturmaßnahmen dieses Kaisers in der Nähe von Sulukulekapı gesucht werden müssen. Eine in der Gegend von Edirnekapı entdeckte Inschrift, die das Datum 1333/34 nennt, bestätigt, daß in diesem Bereich palaiologische Reparaturen stattgefunden haben. ${ }^{747}$ Abgesehen davon kommt ähnliches Mauerwerk auch an der Rückseite des sog. 3. Nebentors (Kalagros-Tor) bei dem Sigma genannten Rücksprung vor. Besonders der aus roten Ziegellagen und weißen Keilsteinen hergestellte Torbogen (Abb. 13) läßt sich mit mehreren palaiologischen Beispielen vergleichen, so z. B. mit Tekfur Sarayı (Abb. 6, 175, 178, 183) oder mit Mermerkule (Abb. 133). Als Beispiele für verwandtes Mauerwerk kann man u.a. die obere Partie von Kurtine 42/43 (Abb. 119), Kurtine 51/52, wo ebenfalls polychrom akzentuierte Bögen vorkommen (Abb. 190), Turm 59 (Abb. 83) sowie den Kurtinenabschnitt zwischen Turm 11 und Yedikulekapısı (Abb. 114, 115)

744 Matschke, Builders, 315-328, präsentierte mehrere Quellen über den Mangel an Baumaterial in palaiologischer Zeit. - Vgl. Anm. 759.

745 Vgl. Anm. 560 und 561.

746 Foss, Fortifications, 59. - Zum Gedicht siehe Meyer-Plath/Schneider, Landmauer, 137, Nr. 48a.

747 Meyer-Plath/Schneider, Landmauer, 138, Nr.48b (Schneider). 
rechnen. Eine Variante mit mehreren Ziegelschichten findet sich auch an der späteren Vermauerung des nördlichen Durchgangs des Goldenen Tors (Abb. 120). ${ }^{748}$

Durch zahlreiche bis vor kurzem in situ erhaltene Inschriften an der Vormauer (Abb. 53, 59, 60) und an der Blachernen-Kurtine 12/13 (Abb. 160, 162) ist man über das geläufige Mauerwerk des 15. Jahrhunderts gut unterrichtet. In dieser Zeit tritt die Ziegelverwendung weitgehend zurück und kommt nur noch gelegentlich in den Stoßfugen vor. Statt dessen dominieren unregelmäßige und unordentlich vermauerte kleinere Quader das Mauerwerk. Das Mauerwerk der Kurtine 12/13 der Blachernen-Mauer, das inschriftlich in das Jahr $1441 \mathrm{zu}$ setzen ist, ${ }^{749}$ zeigt an der Stadtseite auch polychrome Bogenakzentuierung (Abb. 162). Die oberen Teile der Türme 11 und 12 der Komnenen-Mauer stammen eindeutig aus dieser Reparaturphase. Auch hier sind die polychromen Bögen noch zu erkennen (Abb. 24).

Neben der Wiederverwendung von älteren Quadern und Ziegeln begegnen an den Mauern (wie auch an beiden Seemauern) zahlreiche Spolienstücke, ${ }^{750}$ die gewöhnliches Baumaterial ersetzen, zum Teil aber auch dekorativ angebracht worden sind. ${ }^{751}$ Während das Goldene Tor mit zahlreichen wiederverwendeten Stücken dekoriert ist, ${ }^{752}$ kommen in denjenigen Abschnitten der Mauer, die in die Zeit Theodosius' II. datiert werden, kaum Spolien vor. ${ }^{753}$ In den Mauer-

748 Ousterhout, Developements, 78: „I suspect that the blocking of the north arch of the main gate should also be placed in the mid or late fourteenth century."

749 Vgl. Anm. 552.

750 Für die Spolienverwendung in der Spätantike siehe Deichmann, Spolien. Für die Spolienverwendung in Konstantinopel siehe Müller-Wiener, Spoliennutzung, 369-382; Mango, Statuary, 55-75; ders., Spolia, 645-657; ders, Triumphal Way, 173-186; Ousterhout, Master Builders, 140-147.

751 Alle Spolien können hier nicht behandelt werden. Im Katalog (Abschnitt 5.) werden besonders die Stücke, die Dekoration aufweisen, aufgelistet. Außer den von mir aufgenommenen Spolien, wovon einige schon Meyer-Plath/Schneider, Landmauer, 151-152 (Schneider), und Tezcan, Topkapı, 45-49, erwähnt haben, befinden sich mehrere Exemplare heute in Museen, hauptsächlich in den İstanbuler Archäologischen Museen; siehe Fıratlı, Sculpture, 37, Nr.68, Taf.26, Abb.68; vgl. auch Dirimtekin, Haliç, $12-$ 14; ders., Marmara, Abb. 45, 69, 70; Effenberger, Mosesrelief, 237-259; Kalkan/Şahin, Grabstelen, 137-147.

752 Für den Schmuck am Goldenen Tor und die ältere Literatur siehe zuletzt Bardill, Triumphal Arch, 671-696, bes. 681-686; Meyer, Staatsdenkmal, 168-173; Bassett, Urban Image, 212.

753 Die Spoliennutzung scheint eigentlich gerade in dieser Zeit wegen der Schließung der heidnischen Tempel und den damit verbundenen Zerstörungen sonst erheblich zugenommen zu haben, weshalb die Entnahme von Baumaterial aus aufgelassenen Gebäuden wiederholt gesetzlich geregelt werden mußte, siehe Cod. Theod.15, 1, passim. Überblick über die Spolienverwendung in Konstantinopel in frühbyzantinischer Zeit bei Müller-Wiener, Spoliennutzung 369-382, bes.370-371. 
partien, die dem 8. und 9. Jahrhundert zugeschrieben werden können, ersetzen die Spolienstücke hin und wieder Baumaterial. ${ }^{754}$ Das heute kaum erkennbare Senmurw-Relief an Turm 16 (Theophilos-Turm ${ }^{755}$ ) der Blachernen-Mauer aus dem 9. Jahrhundert kann als Beispiel für dekorative Spolienverwendung angesehen werden, die möglicherweise sogar eine apotropäische Funktion beinhaltete. ${ }^{756}$ In den Abschnitten, die seit dem 10. Jahrhundert repariert wurden, begegnet neben willkürlicher auch dekorative Verwendung. ${ }^{757}$ Dazu gehören vor allem die Spolienstücke an den Türmen 83 (Abb. 109, 195, 196) und 84 (Abb. 110, 111), wobei diejenigen an Turm 83 anscheinend erst während der palaiologischen Reparatur ohne Beachtung des Dekors eingesetzt worden sind. Zur „repräsentativen“ Spolienverwendung gehört zweifelsfrei das Rahmenwerk am Vortor des Goldenen Tors (Abb. 7, 8, 185, 205) (siehe Abschnitt 2.1.5.). Auch im 12. Jahrhundert, besonders am Nordtrakt der Komnenen-Mauer im Bereich des Gyrolimne-Tors (Abb. 158, 159a-c), scheinen Spolien ganz bewußt zur Dekoration eingesetzt worden zu sein (siehe Abschnitt 3.4.2.). Obwohl am Südtrakt der Mauer Spolien häufig als gewöhnliches Baumaterial vorkommen, kann die Platte an der feldseitigen Kurtine 8/9 der dekorativen Verwendung zugerechnet werden (Abb. 192). Die zahlreichen Säulenschäfte an Turm 14 der Blachernen-Mauer, die hier Konsolen ersetzen, bestätigen den Bericht von Niketas Choniates, wonach Kaiser Isaakios II. Angelos mehrere noch intakte Gebäude zerstören ließ, um damit neue Bauten zu errichten (Abb. 170). ${ }^{758}$ An

754 Müller-Wiener, Spoliennutzung, 373, äußerte über die Spolienverwendung zwischen dem 7. und 9. Jahrhundert: „Mit Ende der frühbyzantinischen Zeit hörte nach den vorliegenden Nachrichten aber auch der unter den obigen Vorzeichen betriebene Spolienimport nach Konstantinopel auf; das expandierende Wachstum der Stadt hatte mit den großen Pestepidemien seit M. 6 Jh.s ein Ende gefunden. Nach den schweren Angriffen der Perser, Awaren und schließlich der Araber auf Konstantinopel im 7. Jh. begann erst im 8./9 Jahrhundert eine Regenerationsphase, in der freilich die Rolle der Hauptstadt inzwischen so klar war, daß sie keiner weiteren Bestätigung mehr bedurfte. Damit hörte zwar der Abtransport bedeutender Einzelkunstwerke auf (deren Vorrat im Übrigen ja auch nicht unerschöpflich war), es häufen sich dagegen die Fälle ,normaler Spolien-Verwendung' aus einfachen Nützlichkeitsgesichtspunkten."

755 Besonders zur Zeit dieses Kaisers scheint sonst eine große Skrupellosigkeit hinsichtlich des Umgangs mit älteren Bauten geherrscht zu haben. So brachte Theophilos u.a. die Decke des Basiliskos-Hauses in den Lausiakos des Großen Palastes, vgl. Mango, Spolia, 645-646. Zur Spolienverwendung in der Zeit des Theophilos siehe auch MüllerWiener, Spoliennutzung, 373.

756 Vgl. Eyice, Senmurw, 112: „Daß man dieses Symbol gleichzeitig als Schutzmacht angesehen hat, ist nicht sicher. Es liegt jedoch durchaus im Bereich der Möglichkeiten, daß der Steinmetz wie auch der Maurer, der den Turm erbaut hat, dieses Ziel vor Augen hatten." - Für die an beiden Seiten des zugemauerten Blachernen-Tores dekorativ verwendeten Spolien siehe Müller-Wiener, Topographie, 305, Abb. 349.

757 Für die Spolienverwendung im 10. Jahrhundert siehe Mango, Spolia, 645-657.

758 Niketas Choniates, 442/33-444/95; deutsche Übersetzung: Grabler, Abenteurer, 248. 
den in die palaiologische Zeit datierbaren Teilen der Mauer sind neben kleinen Fragmenten, die zumeist gewöhnliches Baumaterial ersetzten, ${ }^{759}$ dekorativ angebrachte Stücke zu finden, so z. B. die mit einem Bogen eingefaßte Platte an Kurtine 51/52 (Abb. 190). Das in dieser Zeit errichtete Mermerkule bzw. Polichnion von Ioannes V. Palaiologos (siehe Abschnitt 2.3.2.) wurde überwiegend aus Spolien mit verbürgter Herkunft errichtet (Abb. 132-134).

Die Spolien kommen an den Mauern als Einzelstücke aus unterschiedlichen Gattungen der Bauplastik als Ganzes oder in Fragmenten vor. Verschiedenen Kapitelltypen ersetzen zumeist nur Quader (Abb. 193, 201, 202) ${ }^{760}$ In mehreren Mauerabschnitten sind Säulenschäfte - in der Regel aus prokonnesischem Marmor, in seltenen Fällen auch aus Porphyr bzw. aus verde antico - teils einzeln, teils in Gruppen oder als Bruchstücke eingefügt. Sie sind zumeist quer in die Wand eingeschoben und dienen als Binder (Abb. 160) ${ }^{761}$ oder - wie oben erwähnt - als Konsolen. Zahlreiche Pfeiler, die sich auf Grund ihrer Maße als Schrankenpfeiler von Templonanlagen bestimmen lassen bzw. zu Pfeilerstellungen gehört haben ${ }^{762}$ wurden zumeist wie übliches Quadermaterial benutzt, treten aber des öfteren in den Eckverbindungen der Türme auf (Abb. 189, 200). Hin und wieder dienen sie auch als Stürze der Licht- bzw. Schießscharten. ${ }^{763}$ Postamente und Basen ersetzen gleichermaßen einfache Quader oder füllen eine später beschädigte Stelle aus (Abb. 7, nördlich des Rahmenwerkes). ${ }^{764}$ Schmucklose oder einige skulptierte Friese, Gesimse sowie Architrave wurden ebenfalls entweder als Binder in die Mauer hineingeschoben oder in Bruchstücken als Quaderersatz verwendet (Abb. 132, 198, 211). Es kommt auch vor, daß zusammengehörige Gesimse bzw. Friese in Stücke zerteilt und im gleichen Mauerabschnitt benutzt wurden (Abb. 195, 196, 206). Sie ersetzen auch hier

759 Matschke, Builders, 327: „One serious problem which further affected construction activity in late Byzantine Constantinople, and perhaps especially so at this time, was the procurement of building materials. Spolia were used often, and probably even predominantly, in both the public and private sector."

760 Zahlreiche großformatige korinthische bzw. komposite Kapitelle sind besonders in den unteren Teilen der Seemauer eingefügt und mit ihrer Oberseite zur Ansicht gebracht. Sie dienten als Verstärkung gegen die Wellen. Aufgrund der Erhöhung des Bodenniveaus sind mehrere heute nicht mehr zu erkennen, siehe Dirimtekin, Marmara, Abb. 41.

761 Größere Ansammlungen befinden sich ebenfalls in den unteren Bereichen der MarmaraSeemauer.

762 Für Beispiele dieser Gattung siehe Peschlow, Didyma, 219-220; ders., Myra, 220-221; ders., Templon, 1449-1475; Ötüken, Kleinasien, 149-151; Hoddinott, Churches, passim; Mathews, Churches, passim.

763 Meistens mit einfachem Soffittenmuster, z. B. am Tekfur Sarayı-Nordturm (als Sturz).

$764 \mathrm{Im}$ Bereich des Bukoleon-Palastes sind ebenfalls zahlreiche Basen wie die Kapitelle in die Mauer eingesetzt, so daß nur die Unterseiten der Plinthen sichtbar sind (Abb. 208). Sie dienten ebenfalls als Mauerverstärkung gegen die Wellen. Für Beispiele dieser Gattung außerhalb Konstantinopels siehe Ötüken, Kleinasien, 166-173. 
nur Baumaterial, so etwa an der feldseitigen Kurtine 5/6 am Südtrakt der Komnenen-Mauer. Einige Gesimse, besonders in den bewußt dekorierten Teilen der Mauer wie dem Rahmenwerk des Vorwerk des Goldenen Tors, behielten auch in ihrer Zweitverwendung ihre gliedernde Funktion bei (Abb. 7, 8). Eine dekorative Verwendung als Sturz einer Schießscharte begegnet an Turm 61 (Abb. 197). Tür- und Fensterrahmen wurden wie andere Bauspolien überall in die Mauern eingefügt (Abb. 186). Die nur bruchstückhaft verwendeten Teile wurden auf unterschiedliche Größen zugeschnitten. Viele davon ragen aus dem Mauerverband hervor und weisen keinerlei Dekoration auf. Im Falle des Kalagros-Tors (Abb. 75) kann davon ausgegangen werden, daß das Türgewände an gleicher Stelle wiederverwendet wurde (siehe Abschnitt 2.2.2.). Auch Platten bzw. Schrankenplatten (Abb. 190, 192) oder Konsolen (Abb. 191), die gelegentlich dekorativ angebracht worden sind (Abb. 191), dienen in verschiedenen Abschnitten der Mauer als Ersatz für Baumaterial (Abb. 194, 207). Im Unterschied zu den am ursprünglichen Anbringungsort befindlichen Reparaturinschriften sind Bruchstücke von solchen Inschriftbändern während späterer Reparaturen (Abb. 187) zum Teil umgekehrt eingemauert worden (Abb. 188). In einigen Fällen können sie bestimmten Kaisern bzw. einer bestimmten Epoche zugeschrieben werden. Darüber hinaus befinden sich an mehreren Abschnitten zahlreiche undeutbare Bruchstücke im Mauerverband. 